\title{
COSET RELATION ALGEBRAS
}

\author{
HAJNAL ANDRÉKA AND STEVEN GIVANT
}

\begin{abstract}
A measurable relation algebra is a relation algebra in which the identity element is a sum of atoms that can be measured in the sense that the "size" of each such atom can be defined in an intuitive and reasonable way (within the framework of the first-order theory of relation algebras). A large class of concrete measurable set relation algebras, using systems of groups and coordinated systems of isomorphisms between quotients of the groups, is constructed in [4. This class of group relation algebras is not large enough to prove that every measurable relation algebra is isomorphic to a group relation algebra and hence is representable.

In the present article, the class of examples of measurable relation algebras is considerably extended by adding one more ingredient to the mix: systems of cosets that are used to "shift" the operation of relative multiplication. It is shown that, under certain additional hypotheses on the system of cosets, each such coset relation algebra with a shifted operation of relative multiplication is an example of a measurable relation algebra. We also show that the class of coset relation algebras does contain examples of measurable relation algebras that are not representable as set relation algebras. In later articles, it will be shown that the class of coset relation algebras is adequate to the task of describing all measurable relation algebras in the sense that every atomic measurable relation algebra is essentially isomorphic to a coset relation algebra (see [6), and the class of group relation algebras is similarly adequate to the task of representing all measurable relation algebras in which the associated groups are finite and cyclic (see [1]). An extended abstract for this series of papers is [5].
\end{abstract}

\section{INTRODUCTION}

In 4, a subidentity element $x$ - that is to say, an element below the identity element - of a relation algebra is defined to be measurable if it is an atom and if the square $x ; 1 ; x$ is a sum of functional elements, that is to say, the sum of elements that satisfy a characteristic property of relations that are functions, namely, that the composition of the converse of the relation with the relation itself is included in the identity relation. The number of non-zero functional elements below the square $x ; 1 ; x$ gives the measure, or the size, of the atom $x$. A relation algebra is said to be measurable if the identity element is the sum of measurable atoms. The group relation algebras constructed in [4 are examples of measurable relation algebras. It turns out, however, that they are not the only examples of measurable relation algebras.

In this paper, a more general class of examples of measurable relation algebras is constructed. The algebras are obtained from group relation algebras by "shifting" the relational composition operation by means of coset multiplication, using

This research was partially supported by Mills College and the Hungarian National Foundation for Scientific Research, Grants T30314 and T35192. 
an auxiliary system of cosets. For that reason, we have called them coset relation algebras. By using this new construction, we show that not all measurable relation algebras are representable. In fact, as hinted in the proof, the class of coset relation algebras includes infinitely many mutually non-isomorphic, non-representable relation algebras. These are new examples of non-representable relation algebras, with a completely different underlying motivation than the examples that have appeared so far in the literature.

These non-representable examples show that it was necessary to broaden the class of group relation algebras, all of which are representable, in order to get a representation theorem for all measurable relation algebras. Indeed, the new class is broad enough for representing all measurable relation algebras, as is shown in [6]. It will be shown in [1] that if the groups $G_{x}$ constructed in an atomic, measurable relation algebra $\mathfrak{A}$ are all finite and cyclic, then $\mathfrak{A}$ is essentially isomorphic to a full group relation algebra. These theorems together provide far-reaching generalizations of the atomic case of Maddux's representation theorem for pair-dense relation algebras in 8 . An extended abstract describing these results and their interconnections was published by the authors in [5]. The reader might find it helpful to consult that article in order to get a overview of the program and its motivation.

In the next section of this paper, the principal results concerning group relation algebras are reviewed. In the third section, a system of shifting cosets is introduced, and a new operation of multiplication is defined with the help of these cosets. Characterizations are given in the fourth section of when the resulting algebra is a measurable relation algebra. A concrete example of such a measurable coset relation algebra that, as it turns out, is not representable, is given in the fifth section. The final section of the paper contains a decomposition theorem for coset relation algebras that is similar to the decomposition theorem for group relation algebras proved in [4. Except for basic facts about groups, this article is intended to be largely self-contained. Readers who wish to learn more about the subject of relation algebras are recommended to look at one or more of the books HirschHodkinson [7, Maddux [9], or Givant [2], 3].

\section{Group Relation algebras}

For the convenience of the reader, here is a summary of the essential notions and results from [4] that will be needed in this paper. Fix a system

$$
G=\left\langle G_{x}: x \in I\right\rangle
$$

of groups $\left\langle G_{x},{ }^{\circ},{ }^{-1}, e_{x}\right\rangle$ that are pairwise disjoint, and an associated system

$$
\varphi=\left\langle\varphi_{x y}:(x, y) \in \mathcal{E}\right\rangle
$$

of quotient isomorphisms. Specifically, we require that $\mathcal{E}$ be an equivalence relation on the index set $I$, and for each pair $(x, y)$ in $\mathcal{E}$, the function $\varphi_{x y}$ be an isomorphism from a quotient group of $G_{x}$ to a quotient group of $G_{y}$. Call

$$
\mathcal{F}=(G, \varphi)
$$

a group pair. The set $I$ is the group index set, and the equivalence relation $\mathcal{E}$ is the (quotient) isomorphism index set, of $\mathcal{F}$. The normal subgroups of $G_{x}$ and $G_{y}$ from which the quotient groups are constructed are uniquely determined by $\varphi_{x y}$, and will be denoted by $H_{x y}$ and $K_{x y}$ respectively, so that $\varphi_{x y}$ maps $G_{x} / H_{x y}$ isomorphically onto $G_{y} / K_{x y}$. 
The elements of the quotient group $G_{x} / H_{x y}$ are cosets, and hence complexes (sets) of group elements. As such they obey the standard laws of group theory. Multiplication of cosets and unions of cosets is an associative operation for which the normal subgroup $H_{x y}$ is the identity element that commutes with every other coset (and every union of cosets). Every coset has an inverse, and the operation of forming inverses of cosets satisfies the first and second involution laws: the inverse of the inverse of a coset is the original coset, and the inverse of the composition of two cosets is the composition of the inverses, in the reverse order.

For a fixed enumeration $\left\langle H_{x y, \gamma}: \gamma<\kappa_{x y}\right\rangle$ (without repetitions) of the cosets of $H_{x y}$ in $G_{x}$, the isomorphism $\varphi_{x y}$ induces a corresponding, or associated, coset system of $K_{x y}$ in $G_{y}$, determined by the rule

$$
K_{x y, \gamma}=\varphi_{x y}\left(H_{x y, \gamma}\right)
$$

for each $\gamma<\kappa_{x y}$. In what follows, it is always assumed that the given coset systems for $H_{x y}$ in $G_{x}$ and for $K_{x y}$ in $G_{y}$ are associated in this manner. Furthermore, it is assumed that the first elements of the coset systems are always the normal subgroups themselves, so that

$$
H_{x y, 0}=H_{x y} \quad \text { and } \quad K_{x y, 0}=K_{x y} .
$$

Definition 2.1. For each pair $(x, y)$ in $\mathcal{E}$ and each $\alpha<\kappa_{x y}$, define a binary relation $R_{x y, \alpha}$ by

$$
R_{x y, \alpha}=\bigcup_{\gamma<\kappa_{x y}} H_{x y, \gamma} \times \varphi_{x y}\left[H_{x y, \gamma} \circ H_{x y, \alpha}\right]=\bigcup_{\gamma<\kappa_{x y}} H_{x y, \gamma} \times\left(K_{x y, \gamma} \circ K_{x y, \alpha}\right) .
$$

Lemma 2.2 (Partition Lemma). The relations $R_{x y, \alpha}$, for $\alpha<\kappa_{x y}$, are non-empty and partition the set $G_{x} \times G_{y}$.

Let $U$ be the union of the disjoint system of groups, and $E$ the equivalence relation on $U$ induced by the isomorphism index set $\mathcal{E}$,

$$
U=\bigcup\left\{G_{x}: x \in I\right\} \quad \text { and } \quad E=\bigcup\left\{G_{x} \times G_{y}:(x, y) \in \mathcal{E}\right\} .
$$

Take $A$ to be the collection of unions of all possible sets of the relations of the form $R_{x y, \alpha}$ for $(x, y)$ in $\mathcal{E}$ and $\alpha<\kappa_{x y}$. It turns out that $A$ is always the universe of a complete and atomic Boolean set algebra.

Theorem 2.3 (Boolean Algebra Theorem). The set $A$ is the universe of a complete, atomic Boolean algebra of subsets of $E$. The atoms in $A$ are the distinct relations $R_{x y, \alpha}$ for $(x, y)$ in $\mathcal{E}$ and $\alpha<\kappa_{x y}$, and the distinct elements in $A$ are the unions of distinct sets of atoms.

The set $A$ does not automatically contain the identity relation $i d_{U}$, so it is important to characterize when $i d_{U}$ does belong to $A$.

Theorem 2.4 (Identity Theorem). For each element $x$ in $I$, the following conditions are equivalent.

(i) The identity relation $i d_{G_{x}}$ on $G_{x}$ is in $A$.

(ii) $R_{x x, 0}=i d_{G_{x}}$.

(iii) $\varphi_{x x}$ is the identity automorphism of $G_{x} /\left\{e_{x}\right\}$.

Consequently, the set $A$ contains the identity relation $i d_{U}$ on the base set $U$ if and only if (iii) holds for each $x$ in I. 
Similarly, the set $A$ is not automatically closed under the operation of converse.

Theorem 2.5 (Converse Theorem). For each pair $(x, y)$ in $\mathcal{E}$, the following conditions are equivalent.

(i) There are an $\alpha<\kappa_{x y}$ and a $\beta<\kappa_{y x}$ such that $R_{x y, \alpha}^{-1}=R_{y x, \beta}$.

(ii) For every $\alpha<\kappa_{x y}$ there is a $\beta<\kappa_{y x}$ such that $R_{x y, \alpha}^{-1}=R_{y x, \beta}$.

(iii) $\varphi_{x y}^{-1}=\varphi_{y x}$.

Moreover, if one of these conditions holds, then we may assume that $\kappa_{y x}=\kappa_{x y}$, and the index $\beta$ in (i) and (ii) is uniquely determined by $H_{x y, \alpha}^{-1}=H_{x y, \beta}$. The set $A$ is closed under converse if and only if (iii) holds for all $(x, y)$ in $\mathcal{E}$.

Convention 2.6. Suppose $A$ is closed under converse. If a pair $(x, y)$ is in $\mathcal{E}$, then $H_{y x}=K_{x y}$, and therefore any coset system for $H_{y x}$ is also a coset system for $K_{x y}$. Since the enumeration $\left\langle H_{y x, \gamma}: \gamma<\kappa_{y x}\right\rangle$ of the cosets of $H_{y x}$ can be freely chosen, we can and always shall choose it so that $\kappa_{y x}=\kappa_{x y}$ and $H_{y x, \gamma}=K_{x y, \gamma}$ for $\gamma<\kappa_{x y}$. It then follows from the Converse Theorem that $K_{y x, \gamma}=H_{x y, \gamma}$ for $\gamma<\kappa_{x y}$.

Finally, the set $A$ is not in general closed under relational composition, except when the composition is empty.

Lemma 2.7. If $(x, y)$ and $(w, z)$ are in $\mathcal{E}$, and if $y \neq w$, then

$$
R_{x y, \alpha} \mid R_{w z, \beta}=\varnothing
$$

for all $\alpha<\kappa_{x y}$ and $\beta<\kappa_{w z}$.

The most important case regarding the composition of two atomic relations is when $y=w$.

Theorem 2.8 (Composition Theorem). For all pairs $(x, y)$ and $(y, z)$ in $\mathcal{E}$, the following conditions are equivalent.

(i) The relation $R_{x y, 0} \mid R_{y z, 0}$ is in $A$.

(ii) For each $\alpha<\kappa_{x y}$ and each $\beta<\kappa_{y z}$, the relation $R_{x y, \alpha} \mid R_{y z, \beta}$ is in $A$.

(iii) For each $\alpha<\kappa_{x y}$ and each $\beta<\kappa_{y z}$,

$$
R_{x y, \alpha} \mid R_{y z, \beta}=\bigcup\left\{R_{x z, \gamma}: H_{x z, \gamma} \subseteq \varphi_{x y}^{-1}\left[K_{x y, \alpha} \circ H_{y z, \beta}\right]\right\} .
$$

(iv) $H_{x z} \subseteq \varphi_{x y}^{-1}\left[K_{x y} \circ H_{y z}\right]$ and $\hat{\varphi}_{x y} \mid \hat{\varphi}_{y z}=\hat{\varphi}_{x z}$, where $\hat{\varphi}_{x y}$ and $\hat{\varphi}_{x z}$ are the mappings induced by $\varphi_{x y}$ and $\varphi_{x z}$ on the quotient of $G_{x}$ modulo the normal subgroup $\varphi_{x y}^{-1}\left[K_{x y}{ }^{\circ} H_{y z}\right]$, while $\hat{\varphi}_{y z}$ is the isomorphism induced by $\varphi_{y z}$ on the quotient of $G_{y}$ modulo the normal subgroup $K_{x y}{ }^{\circ} H_{y z}$.

Consequently, the set $A$ is closed under relational composition if and only if (iv) holds for all pairs $(x, y)$ and $(y, z)$ in $\mathcal{E}$.

Corollary 2.9. If the set $A$ contains the identity relation, then for any pairs $(x, y)$ and $(y, z)$ in $\mathcal{E}$, the following conditions are equivalent.

(i) $R_{x y, \alpha} \mid R_{y z, \beta}$ is in $A$ for some $\alpha<\kappa_{x y}$ and some $\beta<\kappa_{y z}$.

(ii) $R_{x y, \alpha} \mid R_{y z, \beta}$ is in $A$ for all $\alpha<\kappa_{x y}$ and all $\beta<\kappa_{y z}$.

Putting together the preceding theorems yields a characterization, purely in terms of the quotient isomorphisms, of when a group pair gives rise to a complete and atomic set relation algebra. 
Definition 2.10. A group frame is a group pair

$$
\mathcal{F}=\left(\left\langle G_{x}: x \in I\right\rangle,\left\langle\varphi_{x y}:(x, y) \in \mathcal{E}\right\rangle\right)
$$

satisfying the following frame conditions for all pairs $(x, y)$ and $(y, z)$ in $\mathcal{E}$.

(i) $\varphi_{x x}$ is the identity automorphism of $G_{x} /\left\{e_{x}\right\}$ for all $x$.

(ii) $\varphi_{y x}=\varphi_{x y}^{-1}$.

(iii) $\varphi_{x y}\left[H_{x y} \circ H_{x z}\right]=K_{x y} \circ H_{y z}$ and $\varphi_{y z}\left[K_{x y} \circ H_{y z}\right]=K_{x z} \circ K_{y z}$.

(iv) $\hat{\varphi}_{x y} \mid \hat{\varphi}_{y z}=\hat{\varphi}_{x z}$.

Given a group frame $\mathcal{F}$, let $A$ be the collection of all possible unions of relations of the form $R_{x y, \alpha}$ for $(x, y)$ in $\mathcal{E}$ and $\alpha<\kappa_{x y}$. Call $A$ the set of frame relations constructed from $\mathcal{F}$.

Theorem 2.11 (Group Frame Theorem). If $\mathcal{F}$ is a group frame, then the set of frame relations constructed from $\mathcal{F}$ is the universe of a complete, atomic, measurable set relation algebra with base set and unit

$$
U=\bigcup\left\{G_{x}: x \in I\right\} \quad \text { and } \quad E=\bigcup\left\{G_{x} \times G_{y}:(x, y) \in \mathcal{E}\right\}
$$

respectively. The atoms in this algebra are the relations of the form $R_{x y, \alpha}$, and the subidentity atoms are the relations of the form $R_{x x, 0}$. The measure of $R_{x x, 0}$ is just the cardinality of the group $G_{x}$.

The theorem justifies the following definition.

Definition 2.12. Suppose that $\mathcal{F}$ is a group frame. The set relation algebra constructed from $\mathcal{F}$ in Group Frame Theorem 2.11 is called the (full) group relation algebra on $\mathcal{F}$ and is denoted by $\mathfrak{G}[\mathcal{F}]$ (and its universe by $G[\mathcal{F}]$ ). A general group relation algebra is defined to be an algebra that is embeddable into a full group relation algebra.

\section{Coset Systems}

Group relation algebras by themselves are not sufficient to represent all measurable relation algebras as will be seen in Section [5. However, it is shown in [6] that if the operation of composition in a group relation algebra is changed slightly, then the resulting class of new algebras is sufficient to represent all measurable relation algebras. We call these new algebras coset relation algebras.

The operation of relative multiplication in a coset relation algebra is a kind of "shifted" relational composition. To accomplish this shifting, it is necessary to add one more ingredient to a group pair $\mathcal{F}=(G, \varphi)$, namely a system of cosets

$$
\left\langle C_{x y z}:(x, y, z) \in \mathcal{E}_{3}\right\rangle,
$$

where $\mathcal{E}_{3}$ is the set of all triples $(x, y, z)$ such that the pairs $(x, y)$ and $(y, z)$ are in $\mathcal{E}$, and for each such triple, the set $C_{x y z}$ is a coset of the normal subgroup $H_{x y}{ }^{\circ} H_{x z}$ in $G_{x}$. Call the resulting triple

$$
\mathcal{F}=(G, \varphi, C)
$$

a group triple.

Define a new binary multiplication operation $\otimes$ on the pairs of atomic relations in the Boolean algebra $A$ of Theorem 2.3 as follows. 
Definition 3.1. For pairs $(x, y)$ and $(y, z)$ in $\mathcal{E}$, put

$$
R_{x y, \alpha} \otimes R_{y z, \beta}=\bigcup\left\{R_{x z, \gamma}: H_{x z, \gamma} \subseteq \varphi_{x y}^{-1}\left[K_{x y, \alpha} \circ H_{y z, \beta}\right] \circ C_{x y z}\right\}
$$

for all $\alpha<\kappa_{x y}$ and all $\beta<\kappa_{y z}$, and for all other pairs $(x, y)$ and $(w, z)$ in $\mathcal{E}$ with $y \neq w$, put

$$
R_{x y, \alpha} \otimes R_{w z, \beta}=\varnothing
$$

for all $\alpha<\kappa_{x y}$ and $\beta<\kappa_{w z}$. Extend $\otimes$ to all of $A$ by requiring it to distribute over arbitrary unions. This means that for all subsets $X$ and $Y$ of the set of atoms in $A$

$$
(\bigcup X) \otimes(\bigcup Y)=\bigcup\left\{R_{x y, \alpha} \otimes R_{w z, \beta}: R_{x y, \alpha} \in X \text { and } R_{w z, \beta} \in Y\right\}
$$

Comparing the formula defining $R_{x y, \alpha} \otimes R_{y z, \beta}$ in Definition 3.1 with the value of the relational composition $R_{x y, \alpha} \mid R_{y z, \beta}$ given in Composition Theorem 2.8(iii), it is clear that they are very similar in form. In the first case, however, the coset $\varphi_{x y}^{-1}\left[K_{x y, \alpha} \circ H_{y z, \beta}\right]$ of the composite group $H_{x y}{ }^{\circ} H_{x z}$ has been shifted, through coset multiplication by $C_{x y z}$, to another coset of $H_{x y}{ }^{\circ} H_{x z}$, so that in general the value of the $\otimes$-product and the value of relational composition on a given pair of atomic relations will be different, except in certain cases, for example, the case in which the value is the empty set.

Observe that the product $R_{x y, \alpha} \otimes R_{w z, \beta}$ is, by definition, a union of atomic relations in $A$ and is therefore itself a member of $A$. Since $\otimes$ is extended to all of $A$ so as to be completely distributive over unions, and since $A$ is closed under arbitrary unions, it follows that $A$ is automatically closed under the operation $\otimes$. It is not necessary to impose any special conditions on the quotient isomorphisms to ensure this closure, as was the case for relative multiplication in group relation algebras. However, to ensure that $A$ contains the identity relation and is closed under converse, it is still necessary to require conditions (i) and (ii) from Definition 2.10. Conditions (iii) and (iv) in Definition 2.10 ensure that $A$ is closed under relational composition. In order to get a class of algebras large enough to represent all measurable relation algebras, it is necessary to weaken condition (iv), but condition (iii) can be retained. In fact, condition (iv) of Definition 2.10 has to be changed only slightly, as can be seen in Definition 3.2 below.

Every element of a group induces an inner automorphism of the group. In particular, the coset $C_{x y z}$, which is an element of the quotient group

$$
G_{x} /\left(H_{x y} \circ H_{x z}\right),
$$

induces an inner automorphism $\tau_{x y z}$ of the quotient group that is defined by

$$
\tau_{x y z}(D)=C_{x y z}^{-1} \circ D \circ C_{x y z}
$$

for every coset $D$ of $H_{x y}{ }^{\circ} H_{x z}$. This automorphism coincides with the identity automorphism of the quotient group just in case the coset $C_{x y z}$ is in the center of the quotient group, that is to say, just in case

$$
C_{x y z} \circ D=D \circ C_{x y z}
$$

for every coset $D$ of $H_{x y}{ }^{\circ} H_{x z}$.

Definition 3.2. A group triple

$$
\mathcal{F}=(G, \varphi, C)
$$


is a pre-semi-frame if the following three conditions are satisfied.

(i) $\varphi_{x x}$ is the identity automorphism of $G_{x} /\left\{e_{x}\right\}$ for all $x$ in $I$.

(ii) $\varphi_{y x}=\varphi_{x y}^{-1}$ whenever $(x, y)$ is in $\mathcal{E}$.

(iii) $\varphi_{x y}\left[H_{x y} \circ H_{x z}\right]=K_{x y} \circ H_{y z}$ whenever $(x, y, z)$ is in $\mathcal{E}_{3}$.

It is a semi-frame if, in addition, the following fourth condition is also satisfied.

(iv) $\hat{\varphi}_{x y}\left|\hat{\varphi}_{y z}=\tau_{x y z}\right| \hat{\varphi}_{x z}$ whenever $(x, y, z)$ is in $\mathcal{E}_{3}$.

Conditions (i)-(iv) are called the semi-frame conditions.

In condition (iv) of this definition, it is understood that $\hat{\varphi}_{x y}, \hat{\varphi}_{y z}$, and $\hat{\varphi}_{x z}$ are the induced isomorphisms described in Composition Theorem 2.8. They are well defined by semi-frame condition (iii).

If the group triple $\mathcal{F}$ is a pre-semi-frame, then the Boolean set algebra $A$ contains the identity relation on its base set (by Identity Theorem 2.4), and is closed under converse (by Converse Theorem 2.5) and under $\otimes$ (by Definition 3.1). Consequently, it is permissible to form the algebra

$$
\mathfrak{C}[\mathcal{F}]=\left\langle A, \cup, \sim, \otimes,{ }^{-1}, i d_{U}\right\rangle .
$$

Of course, $\mathfrak{C}[\mathcal{F}]$ need not be a relation algebra, that is to say, an abstract algebra of the form

$$
\mathfrak{A}=\left(A,+,-, ;,^{\smile}, 1^{\prime}\right)
$$

in which the following axioms are valid.

(R1) $r+s=s+r$.

(R2) $r+(s+t)=(r+s)+t$.

(R3) $-(-r+s)+-(-r+-s)=s$.

(R4) $r ;(s ; t)=(r ; s) ; t$.

(R5) $r ; 1^{\prime}=r$.

(R6) $r^{\smile \smile=r . ~}$

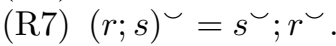

(R8) $(r+s) ; t=r ; t+s ; t$.

(R9) $(r+s)^{\smile}=r^{\smile}+s^{\smile}$.

$(\mathrm{R} 11)(r ; s) \cdot t=0 \quad$ implies $\left(r^{\smile} ; t\right) \cdot s=0$.

(On the basis of the other axioms, (R11) is equivalent to the original law (R10) that Tarski used as the tenth axiom - see, for example, Definition 2.1 in Givant [2]. Consequently, we will not refer to (R10) again.)

Certain relation algebraic axioms are, however, automatically valid in $\mathfrak{C}[\mathcal{F}]$. For example, the Boolean axioms (R1)-(R3) are all valid, because the Boolean part of $\mathfrak{C}[\mathcal{F}]$ is a complete and atomic Boolean set algebra. The first involution law (R6) involves only the operation of converse, so it is valid in $\mathfrak{C}[\mathcal{F}]$. The operation $\otimes$ is distributive over arbitrary unions, as is the operation of converse, so the distributive axioms for relative multiplication and converse over addition, $(\mathrm{R} 8)$ and (R9) respectively, are valid in $\mathfrak{C}[\mathcal{F}]$.

Each of the remaining four axioms, the associative law for relative multiplication (R4), the identity law (R5), the second involution law (R7), and the cycle law (R11) may fail in $\mathfrak{C}[\mathcal{F}]$. It is therefore important to impose conditions on the coset system of a pre-semi-frame that characterize when each of these axioms does hold in $\mathfrak{C}[\mathcal{F}]$. This task is simplified by certain observations. Three of the axioms, namely (R4), (R5), and (R7), are equations, and one of them, namely (R11), is an implication between two equations of the form $\sigma=0$. Each of the equations 
involved is positive in the sense that its terms are constructed from variables and constant symbols using only the operation symbols for addition, multiplication, relative multiplication, and converse. In particular, there is no occurrence of the operation symbol for complement. Each of the axioms is also regular in the sense that no variable occurs more than once on either side of an equation. It is a wellknown result that positive, regular equations, and implications between positive, regular equations of the form $\sigma=0$, hold in an atomic relation algebra (or in any Boolean algebra with completely distributive operators) just in case they hold for all atoms (see, for example, Corollaries 19.26 and 19.28 in Givant [3]). Thus, to verify that any one of these axioms holds in $\mathfrak{C}[\mathcal{F}]$ under certain hypotheses on the coset system, it suffices to verify that it holds for all atomic relations.

We begin with a lemma that says equalities between unions of atomic relations are equivalent to the corresponding coset equalities.

Lemma 3.3. Let $\mathcal{F}$ be a pre-semi-frame, and $(x, y, z)$ a triple in $\mathcal{E}_{3}$. If $D_{0}$ and $D_{1}$ are each unions of cosets of $H_{x y}{ }^{\circ} H_{x z}$, then the following conditions are equivalent.

(i) $D_{0}=D_{1}$.

(ii) $\bigcup\left\{R_{x z, \gamma}: H_{x z, \gamma} \subseteq D_{0}\right\}=\bigcup\left\{R_{x z, \xi}: H_{x z, \xi} \subseteq D_{1}\right\}$.

Proof. Condition (i) obviously implies (ii). To establish the reverse implication, assume $D_{0} \neq D_{1}$. There must then be a coset $M$ of the subgroup $H_{x y}{ }^{\circ} H_{x z}$ that is included in one of the unions, say $D_{0}$, but not the other, $D_{1}$. It follows that $M$ must be disjoint from each of the cosets in $D_{1}$, since two cosets of a subgroup are either equal or disjoint. In particular, each coset $H_{x z, \gamma}$ of $H_{x z}$ that is included in $M$ must be disjoint from $D_{1}$, so the corresponding relation $R_{x z, \gamma}$, which is included in the left-hand side of (ii), by assumption, must be disjoint from the right-hand side of (ii), by Partition Lemma 2.2.

Turn now to the task of finding necessary and sufficient conditions for various relation algebraic laws to hold in the algebra $\mathfrak{C}[\mathcal{F}]$, and begin with the identity law (R5). This law is positive and regular, so it suffices to characterize when it holds for all atomic relations in $\mathfrak{C}[\mathcal{F}]$.

Theorem 3.4 (Identity Law Theorem). Let $\mathcal{F}$ be a pre-semi-frame, and $(x, y)$ a pair in $\mathcal{E}$. The following conditions are equivalent.

(i) $R_{x y, \alpha} \otimes i d_{U}=R_{x y, \alpha}$ for some $\alpha<\kappa_{x y}$.

(ii) $R_{x y, \alpha} \otimes i d_{U}=R_{x y, \alpha}$ for all $\alpha<\kappa_{x y}$.

(iii) $R_{x y, \alpha} \otimes R_{y y, 0}=R_{x y, \alpha}$ for some $\alpha<\kappa_{x y}$.

(iv) $R_{x y, \alpha} \otimes R_{y y, 0}=R_{x y, \alpha}$ for all $\alpha<\kappa_{x y}$.

(v) $C_{x y y}=H_{x y}$.

Consequently, the identity law holds in the algebra $\mathfrak{C}[\mathcal{F}]$ if and only if $(\mathrm{v})$ holds for all pairs $(x, y)$ in $\mathcal{E}$.

Proof. Identity Theorem 2.4 and semi-frame condition (i) imply that

$$
i d_{U}=\bigcup_{w \in I} R_{w w, 0}
$$

Therefore,

$$
R_{x y, \alpha} \otimes i d_{U}=\bigcup_{w \in I} R_{x y, \alpha} \otimes R_{w w, 0}=R_{x y, \alpha} \otimes R_{y y, 0}
$$


by the distributivity of $\otimes$ over arbitrary unions, and the fact that

$$
R_{x y, \alpha} \otimes R_{w w, 0}=\varnothing
$$

whenever $w \neq y$. The equivalences of (i) with (iii), and of (ii) with (iv), are immediate consequences of (11).

We show the equivalence of (iii) and (v), from which it follows trivially that conditions (iii), (iv), and (v) are all equivalent. We have by Definition 3.1, the convention that $H_{y y, 0}=\left\{e_{y}\right\}$, and semi-frame condition (ii) and the convention that $K_{x y, \alpha}=\varphi_{x y}\left(H_{x y, \alpha}\right)$. Now, (iii) holds, by Lemma 3.3 just in case $H_{x y, \alpha}{ }^{\circ} C_{x y y}=$ $H_{x y, \alpha}$, and this last equality holds just in case $C_{x y y}=H_{x y}$, which is just condition (v). This establishes the equivalence of conditions (iii)-(v), and hence of all five conditions, in the statement of the theorem.

The identity law holds in $\mathfrak{C}[\mathcal{F}]$ just in case it holds for all atoms $R_{x y, \alpha}$. Apply the equivalence of (ii) and (v) in the the statement of the theorem to conclude that the identity law holds in $\mathfrak{C}[\mathcal{F}]$ just in case $C_{x y y}=H_{x y}$ for all pairs $(x, y)$ in $\mathcal{E}$.

Take up now the task of characterizing when the cycle law (R11) holds. It suffices to characterize when this implication holds for atoms, and for atoms $r, s$, and $t$, the implication is equivalent to the following atomic form of the cycle law:

$$
s \leq r^{\smile} ; t \quad \text { implies } \quad t \leq r ; s .
$$

Theorem 3.5 (Cycle Law Theorem). Let $\mathcal{F}$ be a pre-semi-frame, and $(x, y, z)$ a triple in $\mathcal{E}_{3}$. The following conditions are equivalent.

(i) If $R_{y z, \beta} \subseteq R_{x y, \alpha}^{-1} \otimes R_{x z, \gamma}$, then $R_{x z, \gamma} \subseteq R_{x y, \alpha} \otimes R_{y z, \beta}$, for some $\alpha<\kappa_{x y}$, $\beta<\kappa_{y z}$, and $\gamma<\kappa_{x z}$.

(ii) If $R_{y z, \beta} \subseteq R_{x y, \alpha}^{-1} \otimes R_{x z, \gamma}$, then $R_{x z, \gamma} \subseteq R_{x y, \alpha} \otimes R_{y z, \beta}$, for all $\alpha<\kappa_{x y}$, $\beta<\kappa_{y z}$, and $\gamma<\kappa_{x z}$.

(iii) $\varphi_{x y}\left[C_{x y z}\right]=C_{y x z}^{-1}$.

Consequently, the cycle law holds in the algebra $\mathfrak{C}[\mathcal{F}]$ just in case (iii) holds for all triples $(x, y, z)$ in $\mathcal{E}_{3}$.

Proof. Fix indices $\alpha<\kappa_{x y}, \beta<\kappa_{y z}$, and $\gamma<\kappa_{x z}$, with the goal of establishing the equivalence of conditions (i) and (iii). Choose $\delta<\kappa_{x y}$ so that

$$
H_{x y, \alpha}^{-1}=H_{x y, \delta},
$$

and observe that

$$
R_{x y, \alpha}^{-1}=R_{y x, \delta},
$$

by semi-frame condition (ii) and Converse Theorem 2.5. Semi-frame condition (ii) and Convention 2.6 imply that

$$
\varphi_{x y}^{-1}=\varphi_{y x}
$$

and

$$
K_{y x, \delta}=H_{x y, \delta}
$$


Combine (11)-(4), and use the definition of $\otimes$, to arrive at

$$
\begin{aligned}
R_{x y, \alpha}^{-1} \otimes R_{x z, \gamma} & =R_{y x, \delta} \otimes R_{x z, \gamma} \\
& =\bigcup\left\{R_{y z, \xi}: H_{y z, \xi} \subseteq \varphi_{y x}^{-1}\left[K_{y x, \delta} \circ H_{x z, \gamma}\right] \circ C_{y x z}\right\} \\
& =\bigcup\left\{R_{y z, \xi}: H_{y z, \xi} \subseteq \varphi_{y x}^{-1}\left[H_{x y, \delta} \circ H_{x z, \gamma}\right]{ }^{\circ} C_{y x z}\right\} \\
& =\bigcup\left\{R_{y z, \xi}: H_{y z, \xi} \subseteq \varphi_{x y}\left[H_{x y, \alpha}^{-1} \circ H_{x z, \gamma}\right]{ }^{\circ} C_{y x z}\right\} .
\end{aligned}
$$

It follows from this string of equalities and Partition Lemma 2.2 that the inclusion

$$
R_{y z, \beta} \subseteq R_{x y, \alpha}^{-1} \otimes R_{x z, \gamma}
$$

is equivalent to the inclusion

$$
H_{y z, \beta} \subseteq \varphi_{x y}\left[H_{x y, \alpha}^{-1} \circ H_{x z, \gamma}\right] \circ C_{y x z} .
$$

A completely analogous argument shows that the inclusion

$$
R_{x z, \gamma} \subseteq R_{x y, \alpha} \otimes R_{y z, \beta}
$$

is equivalent to the inclusion

$$
H_{x z, \gamma} \subseteq \varphi_{x y}^{-1}\left[K_{x y, \alpha} \circ H_{y z, \beta}\right] \circ C_{x y z} .
$$

We now transform (6) in a series of steps. Multiply each side of (6) on the left by the coset $K_{x y, \alpha}$ to obtain the equivalent inclusion

$$
K_{x y, \alpha} \circ H_{y z, \beta} \subseteq K_{x y, \alpha} \circ \varphi_{x y}\left[H_{x y, \alpha}^{-1} \circ H_{x z, \gamma}\right] \circ C_{y x z} .
$$

Notice that the right side of (9) is a coset of $K / H$. (For example, $C_{y x z}$ is a coset of $H_{y x} \circ H_{y z}$, which is equal to $K_{x y}{ }^{\circ} H_{y z}$. Also, $H_{x y, \alpha}^{-1}{ }^{\circ} H_{x z, \gamma}$ is a coset of $H_{x y}{ }^{\circ} H_{x z}$, and $\hat{\varphi}_{x y}$ maps cosets of $H_{x y} \circ H_{x z}$ to cosets of $K / H$, so $\varphi_{x y}\left[H_{x y, \alpha}^{-1}{ }^{\circ} H_{x z, \gamma}\right]$ is a coset of $K_{x y} \circ H_{y z}$. Finally, the product of two cosets of $K / H$ with the coset $K_{x y, \alpha}$ of $K_{x y}$ is again a coset of $K / H$.) The left side of (9) is also a coset of $K / H$. Since two cosets of the same group are either equal or disjoint, the inclusion in (9) is equivalent to the equality

$$
K_{x y, \alpha} \circ H_{y z, \beta}=K_{x y, \alpha}{ }^{\circ} \varphi_{x y}\left[H_{x y, \alpha}^{-1} \circ H_{x z, \gamma}\right] \circ C_{y x z} .
$$

Observe that

$$
\begin{aligned}
K_{x y, \alpha} \circ \varphi_{x y}\left[H_{x y, \alpha}^{-1} \circ H_{x z, \gamma}\right] & =\varphi_{x y}\left[H_{x y, \alpha}\right] \circ \varphi_{x y}\left[H_{x y, \alpha}^{-1} \circ H_{x z, \gamma}\right] \\
& =\varphi_{x y}\left[H_{x y, \alpha} \circ H_{x y, \alpha}^{-1} \circ H_{x z, \gamma}\right] \\
& =\varphi_{x y}\left[H_{x y}{ }^{\circ} H_{x z, \gamma}\right],
\end{aligned}
$$

by the definition of $K_{x y, \alpha}$ (which implies that $\varphi_{x y}\left[H_{x y, \alpha}\right]=K_{x y, \alpha}$ ), the isomorphism properties of $\varphi_{x y}$, and the laws of group theory. Equation (10) can therefore be rewritten in the form

$$
K_{x y, \alpha} \circ H_{y z, \beta}=\varphi_{x y}\left[H_{x y} \circ H_{x z, \gamma}\right] \circ C_{y x z} .
$$

Apply $\varphi_{x y}^{-1}$ to both sides of (11), and use the isomorphism properties of $\varphi_{x y}^{-1}$, to obtain

$$
\begin{aligned}
\varphi_{x y}^{-1}\left[K_{x y, \alpha} \circ H_{y z, \beta}\right] & =\varphi_{x y}^{-1}\left[\varphi_{x y}\left[H_{x y} \circ H_{x z, \gamma}\right] \circ C_{y x z}\right] \\
& =\varphi_{x y}^{-1}\left[\varphi_{x y}\left[H_{x y} \circ H_{x z, \gamma}\right] \circ^{\circ} \varphi_{x y}^{-1}\left[C_{y x z}\right]\right. \\
& =H_{x y} \circ H_{x z, \gamma}{ }^{\circ} \varphi_{x y}^{-1}\left[C_{y x z}\right] .
\end{aligned}
$$


Now $C_{y x z}$ is a coset of $H_{y x} \circ H_{y z}$, which, in turn, is equal to $K_{x y}{ }^{\circ} H_{y z}$, and $\varphi_{x y}$ maps the group $G_{x} /\left(H_{x y} \circ H_{x z}\right)$ isomorphically to the group $G_{y} /\left(K_{x y} \circ H_{y z}\right)$, so the inverse image $\varphi_{x y}^{-1}\left[C_{y x z}\right]$ must be a coset of $H_{x y}{ }^{\circ} H_{x z}$. Consequently,

$$
H_{x y}{ }^{\circ} \varphi_{x y}^{-1}\left[C_{y x z}\right]=\varphi_{x y}^{-1}\left[C_{y x z}\right] \text {, }
$$

so that (12) reduces to

$$
\varphi_{x y}^{-1}\left[K_{x y, \alpha}{ }^{\circ} H_{y z, \beta}\right]=H_{x z, \gamma}{ }^{\circ} \varphi_{x y}^{-1}\left[C_{y x z}\right] .
$$

Summarizing, inclusion (6), and hence also inclusion (5), is equivalent to equation (13).

We now subject equation (8) to similar, but simpler, transformations. Multiply each side of (8) on the right by $C_{x y z}^{-1}$, and use the laws of group theory, to obtain

$$
H_{x z, \gamma}{ }^{\circ} C_{x y z}^{-1} \subseteq \varphi_{x y}^{-1}\left[K_{x y, \alpha}{ }^{\circ} H_{y z, \beta}\right] .
$$

Each side of this inclusion is a coset of $H_{x y}{ }^{\circ} H_{x z}$. Since two cosets of the same group are equal or disjoint, the inclusion in (14) is equivalent to the equation

$$
H_{x z, \gamma}{ }^{\circ} C_{x y z}^{-1}=\varphi_{x y}^{-1}\left[K_{x y, \alpha} \circ H_{y z, \beta}\right] .
$$

Therefore, inclusion (8), and hence also inclusion (7), is equivalent to equation (15).

Combine the results of the last two paragraphs to arrive at the following conclusion: inclusion (5) implies inclusion (7) just in case equation (13) implies equation (15). Compare (13) with (15): the former implies the latter just in case

$$
H_{x z, \gamma}{ }^{\circ} \varphi_{x y}^{-1}\left[C_{y x z}\right]=H_{x z, \gamma}{ }^{\circ} C_{x y z}^{-1},
$$

or, equivalently, just in case

$$
\varphi_{x y}^{-1}\left[C_{y x z}\right]=C_{x y z}^{-1} .
$$

Form the coset inverse of both sides of (16), and apply the isomorphism properties of $\varphi_{x y}^{-1}$, to rewrite (16) as

$$
\varphi_{x y}^{-1}\left[C_{y x z}^{-1}\right]=C_{x y z} .
$$

Apply $\varphi_{x y}$ to both sides of (17) to arrive at the equivalent equation

$$
\varphi_{x y}\left[C_{x y z}\right]=C_{y x z}^{-1} .
$$

It has been shown that the implication from (5) to (7) for fixed $\alpha, \beta$, and $\gamma$, is equivalent to (18). This means that conditions (i) and (iii) in the statement of the theorem are equivalent. Since the formulation of (iii) does not involve any of the three indices $\alpha, \beta$, and $\gamma$, it follows that (iii) implies (i) for each such triple of indices, and hence (iii) implies (ii). The implication from (ii) to (i) is immediate.

The cycle law holds in $\mathfrak{C}[\mathcal{F}]$ just in case it holds for all atoms. Consider such a triple of atoms

$$
R_{x y, \alpha}, \quad R_{w z, \beta}, \quad R_{u v, \gamma},
$$

we want to show

$$
R_{w z, \beta} \subseteq R_{x y, \alpha}^{-1} \otimes R_{u v, \gamma} \quad \text { implies } \quad R_{u v, \gamma} \subseteq R_{x y, \alpha} \otimes R_{w z, \beta} .
$$

If $y=w$ and $u=x$ and $v=z$, then the atomic form of the cycle law holds for the triple just in case $\varphi_{x y}\left[C_{x y z}\right]=C_{y x z}^{-1}$, by the equivalence of conditions (ii) and (iii) in the first part of the theorem. 
Assume $y \neq w$ or $u \neq x$ or $v \neq z$. We show that the law holds trivially, because the left side of the implication reduces to the empty relation. Choose $\xi<\kappa_{x y}$ such that

$$
H_{x y, \alpha}^{-1}=H_{x y, \xi}
$$

and observe that

$$
R_{x y, \alpha}^{-1}=R_{y x, \xi}
$$

by Converse Theorem 2.5. Consequently,

$$
R_{x y, \alpha}^{-1} \otimes R_{u v, \gamma}=R_{y x, \xi} \otimes R_{u v, \gamma} \subseteq G_{y} \times G_{v},
$$

by (19), the definition of $\otimes$, and Partition Lemma 2.2. On the other hand, the relation $R_{w z, \beta}$ is included in $G_{w} \times G_{z}$, by Partition Lemma 2.2. The hypothesis that $w \neq y$ or $z \neq v$ implies that the two Cartesian products

$$
G_{y} \times G_{v} \quad \text { and } \quad G_{w} \times G_{z}
$$

are disjoint, since distinct groups in the given group system are assumed to be disjoint. It follows that

$$
R_{w z, \beta} \cap\left(R_{x y, \alpha}^{-1} \otimes R_{u v, \gamma}\right) \subseteq\left(G_{w} \times G_{z}\right) \cap\left(G_{y} \times G_{v}\right)=\varnothing .
$$

Since $R_{w z, \beta}$ is non-empty, this argument shows that the antecedent of the implication does not hold, so the entire implication must be true. If $u \neq x$, then

$$
R_{x y, \alpha}^{-1} \otimes R_{u v, \gamma}=R_{y x, \xi} \otimes R_{u v, \gamma}=\varnothing,
$$

by (19) and the definition of $\otimes$, so again the antecedent of the asserted implication is false, which means that the entire implication is true.

The next two characterization theorems make use of semi-frame condition (iv). We begin with an auxiliary lemma. Notice that (i) of the lemma coincides with semi-frame condition (iv) stated for the triple $(x, y, z)$ in $\mathcal{E}_{3}$.

Lemma 3.6. Suppose that $\mathcal{F}$ is a pre-semi-frame, and $(x, y, z)$ a triple in $\mathcal{E}_{3}$. The following are equivalent.

(i) If $Q$ is a union of cosets of the subgroup $H_{x y}{ }^{\circ} H_{x z}$ in $G_{x}$, then

$$
\varphi_{y z}\left[\varphi_{x y}[Q]\right]=\varphi_{x z}\left[C_{x y z}^{-1} \circ Q \circ C_{x y z}\right] .
$$

(ii) If $Q$ is a union of cosets of the subgroup $K_{x y}{ }^{\circ} H_{y z}$ in $G_{y}$, then

$$
\varphi_{x z}^{-1}\left[\varphi_{y z}[Q]\right]=C_{x y z}^{-1} \circ \varphi_{x y}^{-1}[Q] \circ C_{x y z} .
$$

(iii) If $Q$ is a union of cosets of the subgroup $K_{x z}{ }^{\circ} K_{y z}$ in $G_{z}$, then

$$
C_{x y z}{ }^{\circ} \varphi_{x z}^{-1}[Q]=\varphi_{x y}^{-1}\left[\varphi_{y z}^{-1}[Q]\right] \circ C_{x y z} .
$$

Proof. Assume (i). To prove (ii), let $Q$ be a union of cosets of $K_{x y}{ }^{\circ} H_{y z}$. By semiframe condition (iii), which holds by the assumption that $\mathcal{F}$ is a pre-semi-frame, we have that $\varphi_{x y}^{-1}[Q]$ is a union of cosets of $H_{x y}{ }^{\circ} H_{x z}$. Substitute $\varphi_{x y}^{-1}[Q]$ in place of $Q$ in (i) to get

$$
\varphi_{y z}\left[\varphi_{x y}\left[\varphi_{x y}^{-1}[Q]\right]\right]=\varphi_{x z}\left[C_{x y z}^{-1} \circ \varphi_{x y}^{-1}[Q] \circ C_{x y z}\right] .
$$

On both sides of (1) there is a union of cosets of $K_{x z}{ }^{\circ} K_{y z}$, again by semi-frame condition (iii). Apply $\varphi_{x z}^{-1}$ to both sides of (i) to obtain

$$
\varphi_{x z}^{-1}\left[\varphi_{y z}\left[\varphi_{x y}\left[\varphi_{x y}^{-1}[Q]\right]\right]\right]=\varphi_{x z}^{-1}\left[\varphi_{x z}\left[C_{x y z}^{-1} \circ \varphi_{x y}^{-1}[Q] \circ C_{x y z}\right]\right] .
$$


Use the inverse property of functions to obtain (ii) from (2). (Notice that the symbol ${ }^{-1}$ is being used two different ways: to denote the inverse functions of the isomorphisms $\varphi_{x y}$ and $\varphi_{x z}$, and to denote the group inverse of the coset $C_{x y z}$. The two different meanings of this particular symbol are standard, and should not cause the reader any confusion.)

In a similar way, to get (iii) from (ii), let $Q$ be a union of cosets of $K_{x z}{ }^{\circ} K_{y z}$. Substitute $\varphi_{y z}^{-1}[Q]$ in place of $Q$ in (ii), multiply both sides by $C_{x y z}$ on the left, and use the inverse property of functions to arrive at (iii).

To get (i) from (iii), let $Q$ be a union of cosets of $H_{x y}{ }^{\circ} H_{x z}$. In (iii), substitute $\varphi_{x z}\left[C_{x y z}^{-1}{ }^{\circ} Q \circ C_{x y z}\right]$ in place of $Q$, and use the inverse property of functions, to get

$$
C_{x y z} \circ C_{x y z}^{-1}{ }^{\circ} Q \circ C_{x y z}=\varphi_{x y}^{-1}\left[\varphi_{y z}^{-1}\left[\varphi_{x z}\left[C_{x y z}^{-1} \circ Q \circ C_{x y z}\right]\right]\right]{ }^{\circ} C_{x y z} .
$$

Multiply both sides with $C_{x y z}^{-1}$ on the right, and use the inverse property for groups to get

$$
Q=\varphi_{x y}^{-1}\left[\varphi_{y z}^{-1}\left[\varphi_{x z}\left[C_{x y z}^{-1} \circ Q \circ C_{x y z}\right]\right]\right] .
$$

Finally, apply $\varphi_{x y}$ and then $\varphi_{y z}$ to both sides of (4) and use the inverse property of functions to get (i) from (4).

Turn next to the second involution law. As before, it suffices to characterize when the equation holds for pairs of atoms in $\mathfrak{C}[\mathcal{F}]$.

Theorem 3.7 (Second Involution Law Theorem). Let $\mathcal{F}$ be a semi-frame, and $(x, y, z)$ a triple in $\mathcal{E}_{3}$. The following conditions are equivalent.

(i) $\left(R_{x y, \alpha} \otimes R_{y z, \beta}\right)^{-1}=R_{y z, \beta}^{-1} \otimes R_{x y, \alpha}^{-1}$ for some $\alpha<\kappa_{x y}$ and some $\beta<\kappa_{y z}$.

(ii) $\left(R_{x y, \alpha} \otimes R_{y z, \beta}\right)^{-1}=R_{y z, \beta}^{-1} \otimes R_{x y, \alpha}^{-1}$ for all $\alpha<\kappa_{x y}$ and all $\beta<\kappa_{y z}$.

(iii) $\varphi_{x z}\left[C_{x y z}\right]=C_{z y x}^{-1}$.

Consequently, the second involution law holds in the algebra $\mathfrak{C}[\mathcal{F}]$ just in case (iii) holds for all triples $(x, y, z)$ in $\mathcal{E}_{3}$.

Proof. Fix $\alpha<\kappa_{x y}$ and $\beta<\kappa_{y z}$, with the goal of showing that conditions (i) and (iii) are equivalent. The first step is to work out concrete formulas for the expressions on the left and right sides of condition (i). The definition of $\otimes$ gives

$$
R_{x y, \alpha} \otimes R_{y z, \beta}=\bigcup\left\{R_{x z, \gamma}: H_{x z, \gamma} \subseteq \varphi_{x y}^{-1}\left[K_{x y, \alpha} \circ H_{y z, \beta}\right]{ }^{\circ} C_{x y z}\right\} .
$$

Form the relational converse of both sides of (10), and apply the distributivity of converse over arbitrary unions, to obtain

$$
\left(R_{x y, \alpha} \otimes R_{y z, \beta}\right)^{-1}=\bigcup\left\{R_{x z, \gamma}^{-1}: H_{x z, \gamma} \subseteq \varphi_{x y}^{-1}\left[K_{x y, \alpha} \circ H_{y z, \beta}\right] \circ C_{x y z}\right\} .
$$

This last equation is equivalent to the equation

$$
\left(R_{x y, \alpha} \otimes R_{y z, \beta}\right)^{-1}=\bigcup\left\{R_{x z, \gamma}^{-1}: H_{x z, \gamma}^{-1} \subseteq\left(\varphi_{x y}^{-1}\left[K_{x y, \alpha}{ }^{\circ} H_{y z, \beta}\right]{ }^{\circ} C_{x y z}\right)^{-1}\right\},
$$

by the first involution law for groups (which says that $\left(g^{-1}\right)^{-1}=g$ for every element $g$ in a group). Converse Theorem 2.5 asserts that

$$
R_{x z, \gamma}^{-1}=R_{z x, \xi} \quad \text { just in case } \quad H_{x z, \gamma}^{-1}=H_{x z, \xi} .
$$

Substitute the right side of each of these equations into the right side of (2) to arrive at

$$
\left(R_{x y, \alpha} \otimes R_{y z, \beta}\right)^{-1}=\bigcup\left\{R_{z x, \xi}: H_{x z, \xi} \subseteq\left(\varphi_{x y}^{-1}\left[K_{x y, \alpha} \circ H_{y z, \beta}\right] \circ C_{x y z}\right)^{-1}\right\} .
$$


Use the second involution law for groups (which says that $(g \circ h)^{-1}=h^{-1} \circ g^{-1}$ for all elements $g$ and $h$ in a group) and the isomorphism properties of $\varphi_{x y}$ to see that

$$
\begin{aligned}
\left(\varphi_{x y}^{-1}\left[K_{x y, \alpha} \circ H_{y z, \beta}\right] \circ C_{x y z}\right)^{-1} & =C_{x y z}^{-1} \circ\left(\varphi_{x y}^{-1}\left[K_{x y, \alpha} \circ H_{y z, \beta}\right]\right)^{-1} \\
& =C_{x y z}^{-1}{ }^{\circ} \varphi_{x y}^{-1}\left[\left(K_{x y, \alpha} \circ H_{y z, \beta}\right)^{-1}\right] \\
& =C_{x y z}^{-1} \circ \varphi_{x y}^{-1}\left[H_{y z, \beta}^{-1} \circ K_{x y, \alpha}^{-1}\right] .
\end{aligned}
$$

Replace the first term by the last term in the right side of (3) to conclude that

$$
\left(R_{x y, \alpha} \otimes R_{y z, \beta}\right)^{-1}=\bigcup\left\{R_{z x, \xi}: H_{x z, \xi} \subseteq C_{x y z}^{-1} \circ \varphi_{x y}^{-1}\left[H_{y z, \beta}^{-1} \circ K_{x y, \alpha}^{-1}\right]\right\} .
$$

The next step is to work out an analogous expression for the right side of (i). Choose $\rho<\kappa_{x y}$ and $\eta<\kappa_{y z}$ so that

$$
K_{x y, \alpha}^{-1}=K_{x y, \rho} \quad \text { and } \quad H_{y z, \beta}^{-1}=H_{y z, \eta} .
$$

Apply semi-frame condition (ii) and Converse Theorem 2.5 to obtain

$$
R_{x y, \alpha}^{-1}=R_{y x, \rho} \quad \text { and } \quad R_{y z, \beta}^{-1}=R_{z y, \eta} .
$$

Use (6) and the definition of $\otimes$ to get

$$
\begin{aligned}
R_{y z, \beta}^{-1} \otimes R_{x y, \alpha}^{-1} & =R_{z y, \eta} \otimes R_{y x, \rho} \\
& =\bigcup\left\{R_{z x, \gamma}: H_{z x, \gamma} \subseteq \varphi_{z y}^{-1}\left[K_{z y, \eta} \circ H_{y x, \rho}\right] \circ C_{z y x}\right\} .
\end{aligned}
$$

Convention 2.6 and (5) yield

$$
K_{z y, \eta}=H_{y z, \eta}=H_{y z, \beta}^{-1} \quad \text { and } \quad H_{y x, \rho}=K_{x y, \rho}=K_{x y, \alpha}^{-1} .
$$

Combine (7) and (8) to arrive at

$$
R_{y z, \beta}^{-1} \otimes R_{x y, \alpha}^{-1}=\bigcup\left\{R_{z x, \gamma}: H_{z x, \gamma} \subseteq \varphi_{z y}^{-1}\left[H_{y z, \beta}^{-1}{ }^{\circ} K_{x y, \alpha}^{-1}\right]{ }^{\circ} C_{z y x}\right\} .
$$

Apply the isomorphism $\varphi_{z x}$ to both sides of the inclusion

$$
H_{z x, \gamma} \subseteq \varphi_{z y}^{-1}\left[H_{y z, \beta}^{-1} \circ K_{x y, \alpha}^{-1}\right]{ }^{\circ} C_{z y x}
$$

to obtain the equivalent inclusion

$$
\varphi_{z x}\left[H_{z x, \gamma}\right] \subseteq \varphi_{z x}\left[\varphi_{z y}^{-1}\left[H_{y z, \beta}^{-1} \circ K_{x y, \alpha}^{-1}\right] \circ C_{z y x}\right] .
$$

Use the definition of the coset $K_{z x, \gamma}$ as the image of the coset $H_{z x, \gamma}$ under the isomorphism $\varphi_{z x}$, and then use Convention 2.6. to rewrite the left side of (11) as

$$
\varphi_{z x}\left[H_{z x, \gamma}\right]=K_{z x, \gamma}=H_{x z, \gamma} .
$$

The right side of (11) may also be rewritten in the following way:

$$
\begin{aligned}
\varphi_{z x}\left[\varphi_{z y}^{-1}\left[H_{y z, \beta}^{-1} \circ K_{x y, \alpha}^{-1}\right] \circ C_{z y x}\right] & =\varphi_{z x}\left[\varphi_{z y}^{-1}\left[H_{y z, \beta}^{-1} \circ K_{x y, \alpha}^{-1}\right]\right] \circ \varphi_{z x}\left[C_{z y x}\right] \\
& =\varphi_{x z}^{-1}\left[\varphi_{y z}\left[H_{y z, \beta}^{-1} \circ K_{x y, \alpha}^{-1}\right]\right] \circ \varphi_{z x}\left[C_{z y x}\right] \\
& =C_{x y z}^{-1} \circ \varphi_{x y}^{-1}\left[H_{y z, \beta}^{-1} \circ K_{x y, \alpha}^{-1}\right] \circ C_{x y z}{ }^{\circ} \varphi_{z x}\left[C_{z y x}\right] .
\end{aligned}
$$

The first equality uses the isomorphism property of $\varphi_{z x}$, the second uses semi-frame condition (ii) which says that

$$
\varphi_{z x}=\varphi_{x z}^{-1} \quad \text { and } \quad \varphi_{y z}=\varphi_{z y}^{-1},
$$


and the third equality uses Lemma 3.6(ii) (with $H_{y z, \beta}^{-1} \circ K_{x y, \alpha}^{-1}$ in place of $Q$ ). Combine (12) with (13) to conclude that the inclusion in (11), and consequently also the one in (10), is equivalent to the inclusion

$$
H_{x z, \gamma} \subseteq C_{x y z}^{-1} \circ \varphi_{x y}^{-1}\left[H_{y z, \beta}^{-1} \circ K_{x y, \alpha}^{-1}\right] \circ C_{x y z} \circ \varphi_{z x}\left[C_{z y x}\right] .
$$

Use the equivalence between (10) and (14) to rewrite (9) as

$$
\begin{aligned}
& R_{y z, \beta}^{-1} \otimes R_{x y, \alpha}^{-1}= \\
& \qquad\left\{R_{z x, \gamma}: H_{x z, \gamma} \subseteq C_{x y z}^{-1} \circ \varphi_{x y}^{-1}\left[H_{y z, \beta}^{-1} \circ K_{x y, \alpha}^{-1}\right] \circ C_{x y z} \circ \varphi_{z x}\left[C_{z y x}\right]\right\} .
\end{aligned}
$$

It follows from (4) and (15) that the equation in (i) holds just in case the right side of (4) is equal to the right side of (15). The right sides of (4) and (15) are equal just in case the cosets

$$
C_{x y z}^{-1} \circ \varphi_{x y}^{-1}\left[H_{y z, \beta}^{-1} \circ K_{x y, \alpha}^{-1}\right]
$$

and

$$
C_{x y z}^{-1} \circ \varphi_{x y}^{-1}\left[H_{y z, \beta}^{-1} \circ K_{x y, \alpha}^{-1}\right] \circ C_{x y z}{ }^{\circ} \varphi_{z x}\left[C_{z y x}\right]
$$

are equal, by Lemma 3.3. (Notice that (16) and (17) really are cosets of $H_{x y} \circ H_{x z}$. In more detail, each of the factors in (16) and (17) is a coset of $H_{x y}{ }^{\circ} H_{x z}$, so the composition of these factors is also a coset of $H_{x y}{ }^{\circ} H_{x z}$. For example, $H_{y z, \beta}^{-1}{ }^{\circ} K_{x y, \alpha}^{-1}$ is a coset of $K_{x y}{ }^{\circ} H_{y z}$, and $\hat{\varphi}_{x y}$ maps the group $G_{x} /\left(H_{x y} \circ H_{x z}\right)$ isomorphically onto the group $G_{y} /(K / H)$, so the inverse image $\varphi_{x y}^{-1}\left[H_{y z, \beta}^{-1}{ }^{\circ} K_{x y, \alpha}^{-1}\right]$ must be a coset of $H_{x y} \circ H_{x z}$. The isomorphism $\hat{\varphi}_{z x}$, which coincides with $\hat{\varphi}_{x z}^{-1}$, maps the group $G_{z} /(K / K)$ isomorphically onto the group $G_{x} /\left(H_{x y}{ }^{\circ} H_{x z}\right)$, and $C_{z y x}$ is a coset of $H_{z y} \circ H_{z x}=K / K$, so the image $\varphi_{z x}\left[C_{z y x}\right]$ must be a coset of $H_{x y}{ }^{\circ} H_{x z}$.) The cosets in (16) and (17) are equal just in case

$$
H_{x y} \circ H_{x z}=C_{x y z}{ }^{\circ} \varphi_{z x}\left[C_{z y x}\right]
$$

or, put another way, they are equal just in case

$$
\varphi_{z x}\left[C_{z y x}\right]^{-1}=C_{x y z}
$$

by the cancellation law for groups. Rewrite (18) as

$$
\varphi_{z x}\left[C_{z y x}^{-1}\right]=C_{x y z},
$$

using the isomorphism properties of $\varphi_{z x}$, and then apply the inverse $\varphi_{x z}$ of the isomorphism $\varphi_{z x}$ to both sides of (19) to obtain the equivalent equation

$$
C_{z y x}^{-1}=\varphi_{x z}\left[C_{x y z}\right] .
$$

Combine these various equivalences to conclude that (i) holds if and only if (20) holds, that is to say, if and only if (iii) holds.

It has been shown that (i) and (iii) are equivalent for any fixed $\alpha$ and $\beta$. Since (iii) does not involve $\alpha$ and $\beta$, it may be concluded that (iii) implies (i) for any $\alpha$ and $\beta$, and hence (iii) implies (ii). The implication from (ii) to (i) is trivial.

The form of the second involution law as a positive, regular equation implies that it holds in $\mathfrak{C}[\mathcal{F}]$ just in case it holds for all atoms $R_{x y, \alpha}$ and $R_{w z, \beta}$ in $\mathfrak{C}[\mathcal{F}]$. If $y=w$, then the law holds for the given pair of atoms just in case $\varphi_{x z}\left[C_{x y z}\right]=C_{z y x}^{-1}$, by the equivalence of conditions (ii) and (iii) established above. 
Assume $y \neq w$. We show that the second involution law holds automatically for the given pair of atoms. Indeed, choose $\gamma$ and $\delta$ so that

$$
H_{x y, \alpha}^{-1}=H_{x y, \gamma} \quad \text { and } \quad H_{w z, \beta}^{-1}=H_{w z, \delta} .
$$

Semi-frame condition (ii) and Converse Theorem 2.5 imply that

$$
R_{x y, \alpha}^{-1}=R_{y x, \gamma} \quad \text { and } \quad R_{w z, \beta}^{-1}=R_{z w, \delta} .
$$

Combine this with the definition of $\otimes$ under the assumption that $y \neq w$ to obtain

$$
R_{w z, \beta}^{-1} \otimes R_{x y, \alpha}^{-1}=R_{z w, \delta} \otimes R_{y z, \gamma}=\varnothing
$$

and

$$
\left(R_{x y, \alpha} \otimes R_{w z, \beta}\right)^{-1}=\varnothing^{-1}=\varnothing .
$$

Since the right sides of (21) and (22) are equal, so are the left sides.

Turn finally to the task of characterizing when the associative law for relative multiplication holds in an algebra $\mathfrak{C}[\mathcal{F}]$. Again, it suffices to characterize when it holds for atoms. It is helpful to introduce a bit of notation. Let $\mathcal{E}_{4}$ denote the set of quadruples $(x, y, z, w)$ such that the pairs $(x, y),(x, z)$, and $(x, w)$ are all in $\mathcal{E}$, or, equivalently, such that the triples $(x, y, z)$ and $(x, z, w)$ are in $\mathcal{E}_{3}$.

Theorem 3.8 (Associative Law Theorem). Let $\mathcal{F}$ be a semi-frame, and $(x, y, z, w)$ a quadruple in $\mathcal{E}_{4}$. The following conditions are equivalent.

(i) $\left(R_{x y, \alpha} \otimes R_{y z, \beta}\right) \otimes R_{z w, \gamma}=R_{x y, \alpha} \otimes\left(R_{y z, \beta} \otimes R_{z w, \gamma}\right)$ for some $\alpha<\kappa_{x y}$, $\beta<\kappa_{y z}$ and $\gamma<\kappa_{z w}$.

(ii) $\left(R_{x y, \alpha} \otimes R_{y z, \beta}\right) \otimes R_{z w, \gamma}=R_{x y, \alpha} \otimes\left(R_{y z, \beta} \otimes R_{z w, \gamma}\right)$ for all $\alpha<\kappa_{x y}, \beta<\kappa_{y z}$ and $\gamma<\kappa_{z w}$.

(iii) $C_{x y z}{ }^{\circ} C_{x z w}=\varphi_{y x}\left[C_{y z w}{ }^{\circ} H_{y x}\right] \circ C_{x y w}$.

Consequently, the associative law for $\otimes$ holds in the algebra $\mathfrak{C}[\mathcal{F}]$ just in case (iii) holds for all quadruples $(x, y, z, w)$ in $\mathcal{E}_{4}$.

Proof. Fix some $\alpha<\kappa_{x y}, \beta<\kappa_{y z}$, and $\gamma<\kappa_{z w}$, with goal of establishing the equivalence of (i) and (iii). The first task is to compute and simplify an expression for

$$
\left(R_{x y, \alpha} \otimes R_{y z, \beta}\right) \otimes R_{z w, \gamma} .
$$

The definition of $\otimes$ implies that

$$
R_{x y, \alpha} \otimes R_{y z, \beta}=\bigcup\left\{R_{x z, \xi}: H_{x z, \xi} \subseteq \varphi_{x y}^{-1}\left[K_{x y, \alpha} \circ H_{y z, \beta}\right] \circ C_{x y z}\right\} .
$$

Form the product, in the sense of $\otimes$, on both sides of (2) on the right with $R_{z w, \gamma}$, and use the distributivity of $\otimes$ over arbitrary unions, to see that (11) is equal to the union

$$
\bigcup\left\{R_{x z, \xi} \otimes R_{z w, \gamma}: H_{x z, \xi} \subseteq \varphi_{x y}^{-1}\left[K_{x y, \alpha} \circ H_{y z, \beta}\right]{ }^{\circ} C_{x y z}\right\} .
$$

The definition of $\otimes$ also yields

$$
R_{x z, \xi} \otimes R_{z w, \gamma}=\bigcup\left\{R_{x w, \rho}: H_{x w, \rho} \subseteq \varphi_{x z}^{-1}\left[K_{x z, \xi}{ }^{\circ} H_{z w, \gamma}\right]{ }^{\circ} C_{x z w}\right\}
$$

for each $\xi$. Write

$$
D_{1}=\varphi_{x y}^{-1}\left[K_{x y, \alpha} \circ H_{y z, \beta}\right] \circ C_{x y z},
$$


and observe that $D_{1}$ is a coset of the normal subgroup $H_{x y}{ }^{\circ} H_{x z}$ in $G_{x}$. Combine (5) with (3) and (4) to arrive at the equality of (1) with

$$
\bigcup\left\{R_{x w, \rho}: H_{x w, \rho} \subseteq \varphi_{x z}^{-1}\left[K_{x z, \xi}{ }^{\circ} H_{z w, \gamma}\right]{ }^{\circ} C_{x z w} \text { for some } H_{x z, \xi} \subseteq D_{1}\right\} .
$$

This union may be rewritten as

$$
\bigcup\left\{R_{x w, \rho}: H_{x w, \rho} \subseteq \bigcup\left\{\varphi_{x z}^{-1}\left[K_{x z, \xi}{ }^{\circ} H_{z w, \gamma}\right]{ }^{\circ} C_{x z w}: H_{x z, \xi} \subseteq D_{1}\right\}\right\} .
$$

In more detail, the sets

$$
\varphi_{x z}^{-1}\left[K_{x z, \xi}{ }^{\circ} H_{z w, \gamma}\right]{ }^{\circ} C_{x z w},
$$

for various $\xi$, are cosets of $H_{x z}{ }^{\circ} H_{x w}$ (since $\varphi_{x z}$ induces an isomorphism from $G_{x} /\left(H_{x z}{ }^{\circ} H_{x w}\right)$ to $\left.G_{z} /\left(K_{x z} \circ H_{z w}\right)\right)$, and any coset $H_{x w, \rho}$ of $H_{x w}$ that is contained in a union of cosets of $H_{x z}{ }^{\circ} H_{x w}$ must be contained entirely within one of these cosets. It follows that (11) and (6) are equal.

We now transform (6) in a series of steps. First,

$$
\begin{aligned}
\bigcup\left\{K_{x z, \xi}: H_{x z, \xi} \subseteq D_{1}\right\} & =\bigcup\left\{\varphi_{x z}\left[H_{x z, \xi}\right]: H_{x z, \xi} \subseteq D_{1}\right\} \\
& =\varphi_{x z}\left[\bigcup\left\{H_{x z, \xi}: H_{x z, \xi} \subseteq D_{1}\right\}\right] \\
& =\varphi_{x z}\left[D_{1}\right],
\end{aligned}
$$

by the definition of $K_{x z, \xi}$ as the image of $H_{x z, \xi}$ under the mapping $\varphi_{x z}$, the distributivity of function images over unions, and the fact that $D_{1}$ is the union of the set of cosets of $H_{x z}$ that are included in it, by (5) and the remark following (5). Therefore

$$
\begin{aligned}
\bigcup\left\{\varphi_{x z}^{-1}\left[K_{x z, \xi} H_{z w, \gamma}\right] \circ\right. & \left.C_{x z w}: H_{x z, \xi} \subseteq D_{1}\right\} \\
& =\bigcup\left\{\varphi_{x z}^{-1}\left[K_{x z, \xi} \circ H_{z w, \gamma}\right]: H_{x z, \xi} \subseteq D_{1}\right\} \circ C_{x z w} \\
& =\varphi_{x z}^{-1}\left[\bigcup\left\{K_{x z, \xi} H_{z w, \gamma}: H_{x z, \xi} \subseteq D_{1}\right\}\right] \circ C_{x z w} \\
& =\varphi_{x z}^{-1}\left[\bigcup\left\{K_{x z, \xi} \circ K_{x z} \circ H_{z w, \gamma}: H_{x z, \xi} \subseteq D_{1}\right\}\right]{ }^{\circ} C_{x z w} \\
& =\varphi_{x z}^{-1}\left[\bigcup\left\{K_{x z, \xi}: H_{x z, \xi} \subseteq D_{1}\right\} \circ K_{x z} \circ H_{z w, \gamma}\right] \circ C_{x z w} \\
& =\varphi_{x z}^{-1}\left[\varphi_{x z}\left[D_{1}\right] \circ K_{x z} \circ H_{z w, \gamma}\right] \circ C_{x z w} \\
& =\varphi_{x z}^{-1}\left[\varphi_{x z}\left[D_{1}\right]\right]{ }^{\circ} \varphi_{x z}^{-1}\left[K_{x z} \circ H_{z w, \gamma}\right]{ }^{\circ} C_{x z w} \\
& =D_{1} \circ \varphi_{x z}^{-1}\left[K_{x z} \circ H_{z w, \gamma}\right] \circ C_{x z w} \\
& =\varphi_{x y}^{-1}\left[K_{x y, \alpha} \circ H_{y z, \beta}\right]{ }^{\circ} C_{x y z}{ }^{\circ} \varphi_{x z}^{-1}\left[K_{x z} \circ H_{z w, \gamma}\right]{ }^{\circ} C_{x z w},
\end{aligned}
$$

by the distributivity of coset composition over arbitrary unions, the distributivity of inverse function images over arbitrary unions, the fact that $K_{x z}$ is the identity element for its group of cosets, the distributivity of coset composition over arbitrary unions, (77), the isomorphism property of $\varphi_{x z}^{-1}$, the fact that $\varphi_{x z}$ and $\varphi_{x z}^{-1}$ are inverses of one another (by semi-frame condition (ii)), and the definition of $D_{1}$ in (5).

Recall that $C_{x y z}$ is a coset of $H_{x y}{ }^{\circ} H_{x z}$. The latter is the identity element of the quotient group $G_{x} /\left(H_{x y} \circ H_{x z}\right)$, and also the image of $K / K$ under the inverse 
isomorphism $\varphi_{x z}^{-1}$. Consequently,

$$
\begin{aligned}
C_{x y z} \circ \varphi_{x z}^{-1}\left[K_{x z} \circ H_{z w, \gamma}\right] & =C_{x y z} \circ H_{x y} \circ H_{x z} \circ \varphi_{x z}^{-1}\left[K_{x z} \circ H_{z w, \gamma}\right] \\
& =C_{x y z}{ }^{\circ} \varphi_{x z}^{-1}[K / K] \circ \varphi_{x z}^{-1}\left[K_{x z} \circ H_{z w, \gamma}\right] \\
& =C_{x y z}{ }^{\circ} \varphi_{x z}^{-1}\left[K / K \circ K_{x z} \circ H_{z w, \gamma}\right] \\
& =C_{x y z}{ }^{\circ} \varphi_{x z}^{-1}\left[K / K \circ H_{z w, \gamma}\right] \\
& =C_{x y z}{ }^{\circ} \varphi_{x z}^{-1}\left[K / K \circ K_{y z} \circ H_{z w, \gamma}\right] \\
& =\varphi_{x y}^{-1}\left[\varphi_{y z}^{-1}\left[K / K \circ K_{y z} \circ H_{z w, \gamma}\right]\right]{ }^{\circ} C_{x y z} \\
& =\varphi_{x y}^{-1}\left[\varphi_{y z}^{-1}[K / K] \circ \varphi_{y z}^{-1}\left[K_{y z}{ }^{\circ} H_{z w, \gamma}\right]\right] \circ C_{x y z} \\
& =\varphi_{x y}^{-1}\left[K / H \circ \varphi_{y z}^{-1}\left[K_{y z} \circ H_{z w, \gamma}\right]{ }^{\circ} C_{x y z} .\right.
\end{aligned}
$$

The sixth equality uses Lemma 3.6(iii) (with $K / K \circ K_{y z} \circ H_{z w, \gamma}$ in place of $Q$ ), the seventh the isomorphism property of $\varphi_{y z}^{-1}$, and the eighth the fact that $\varphi_{y z}$ maps $K / H$ to $K / K$.

Combine the last two strings of equalities with the isomorphism properties of $\varphi_{x y}^{-1}$, and the fact that $K_{x y}{ }^{\circ} H_{y z}$ is the identity element of the quotient group $G_{y} /\left(K_{x y} \circ H_{y z}\right)$, to arrive at

$$
\begin{aligned}
& \bigcup\left\{\varphi_{x z}^{-1}\left[K_{x z, \xi} \circ H_{z w, \gamma}\right]{ }^{\circ} C_{x z w}: H_{x z, \xi} \subseteq D_{1}\right\} \\
&=\varphi_{x y}^{-1}\left[K_{x y, \alpha} \circ H_{y z, \beta}\right]{ }^{\circ} C_{x y z}{ }^{\circ} \varphi_{x z}^{-1}\left[K_{x z} \circ H_{z w, \gamma}\right] \circ C_{x z w} \\
&=\varphi_{x y}^{-1}\left[K_{x y, \alpha} \circ H_{y z, \beta}\right]{ }^{\circ} \varphi_{x y}^{-1}\left[K / H \circ \varphi_{y z}^{-1}\left[K_{y z}{ }^{\circ} H_{z w, \gamma}\right]\right]{ }^{\circ} C_{x y z} \circ C_{x z w} \\
&=\varphi_{x y}^{-1}\left[K_{x y, \alpha}{ }^{\circ} H_{y z, \beta} \circ K / H \circ \varphi_{y z}^{-1}\left[K_{y z}{ }^{\circ} H_{z w, \gamma}\right]\right] \circ C_{x y z}{ }^{\circ} C_{x z w} \\
&=\varphi_{x y}^{-1}\left[K_{x y, \alpha} \circ H_{y z, \beta}{ }^{\circ} \varphi_{y z}^{-1}\left[K_{y z}{ }^{\circ} H_{z w, \gamma}\right]\right] \circ C_{x y z}{ }^{\circ} C_{x z w} .
\end{aligned}
$$

Conclusion: (6) may be rewritten as the inclusion

$$
\bigcup\left\{R_{x w, \rho}: H_{x w, \rho} \subseteq \varphi_{x y}^{-1}\left[K_{x y, \alpha}{ }^{\circ} H_{y z, \beta}{ }^{\circ} \varphi_{y z}^{-1}\left[K_{y z} \circ H_{z w, \gamma}\right]\right]{ }^{\circ} C_{x y z}{ }^{\circ} C_{x z w}\right\},
$$

so (1) and (8) are equal.

The next task is to work out an analogous expression for

$$
R_{x y, \alpha} \otimes\left(R_{y z, \beta} \otimes R_{z w, \gamma}\right)
$$

in an analogous fashion. Write

$$
D_{2}=\varphi_{y z}^{-1}\left[K_{y z, \beta} \circ H_{z w, \gamma}\right] \circ C_{y z w} .
$$

The definition of $\otimes$ and (10) imply that

$$
R_{y z, \beta} \otimes R_{z w, \gamma}=\bigcup\left\{R_{y w, \xi}: H_{y w, \xi} \subseteq D_{2}\right\} .
$$

Form the $\otimes$ product, on both sides of this equation on the left with $R_{x y, \alpha}$, and use the distributivity of $\otimes$ over arbitrary unions, to see that (9) is equal to

$$
\bigcup\left\{R_{x y, \alpha} \otimes R_{y w, \xi}: H_{y w, \xi} \subseteq D_{2}\right\} .
$$

Since

$$
R_{x y, \alpha} \otimes R_{y w, \xi}=\bigcup\left\{R_{x w, \rho}: H_{x w, \rho} \subseteq \varphi_{x y}^{-1}\left[K_{x y, \alpha} \circ H_{y w, \xi}\right]{ }^{\circ} C_{x y w}\right\},
$$

by the definition of $\otimes$, it follows that (12), and hence also (9), is equal to

$$
\bigcup\left\{R_{x w, \rho}: H_{x w, \rho} \subseteq \varphi_{x y}^{-1}\left[K_{x y, \alpha}{ }^{\circ} H_{y w, \xi}\right]{ }^{\circ} C_{x y w} \text { for some } H_{y w, \xi} \subseteq D_{2}\right\} .
$$


This union can be rewritten as

$$
\bigcup\left\{R_{x w, \rho}: H_{x w, \rho} \subseteq \bigcup\left\{\varphi_{x y}^{-1}\left[K_{x y, \alpha} \circ H_{y w, \xi}\right] \circ C_{x y w}: H_{y w, \xi} \subseteq D_{2}\right\}\right\},
$$

and therefore also as

$$
\bigcup\left\{R_{x w, \rho}: H_{x w, \rho} \subseteq \varphi_{x y}^{-1}\left[K_{x y, \alpha}{ }^{\circ} D_{2}\right] \circ C_{x y w}\right\} .
$$

(This last step uses the distributivity of coset compositions and of inverse function images over arbitrary unions.) Use the identity element property for $K_{y z}$ with respect to its cosets, the isomorphism properties of $\varphi_{y z}^{-1}$ on cosets and unions of cosets of $K_{y z}$, and the definition of $K_{y z, \beta}$ to write

$$
\begin{aligned}
\varphi_{y z}^{-1}\left[K_{y z, \beta} \circ H_{z w, \gamma}\right] & =\varphi_{y z}^{-1}\left[K_{y z, \beta} \circ K_{y z} \circ H_{z w, \gamma}\right] \\
& =\varphi_{y z}^{-1}\left[K_{y z, \beta} \circ^{\circ} \varphi_{y z}^{-1}\left[K_{y z} \circ H_{z w, \gamma}\right]\right. \\
& =H_{y z, \beta} \circ \varphi_{y z}^{-1}\left[K_{y z} \circ H_{z w, \gamma}\right] .
\end{aligned}
$$

It follows that

$$
\begin{aligned}
& \varphi_{x y}^{-1}\left[K_{x y, \alpha} \circ D_{2}\right] \circ C_{x y w} \\
&=\varphi_{x y}^{-1}\left[K_{x y, \alpha} \circ \varphi_{y z}^{-1}\left[K_{y z, \beta} \circ H_{z w, \gamma}\right] \circ C_{y z w}\right] \circ C_{x y w} \\
&=\varphi_{x y}^{-1}\left[K_{x y, \alpha} \circ H_{y z, \beta} \circ \varphi_{y z}^{-1}\left[K_{y z} \circ H_{z w, \gamma}\right] \circ C_{y z w}\right] \circ C_{x y w} \\
&=\varphi_{x y}^{-1}\left[K_{x y, \alpha} \circ K_{x y} \circ H_{y z, \beta} \circ \varphi_{y z}^{-1}\left[K_{y z} \circ H_{z w, \gamma}\right] \circ C_{y z w}\right]{ }^{\circ} C_{x y w} \\
&=\varphi_{x y}^{-1}\left[K_{x y, \alpha} \circ H_{y z, \beta} \circ \varphi_{y z}^{-1}\left[K_{y z} \circ H_{z w, \gamma}\right] \circ K_{x y}{ }^{\circ} C_{y z w}\right] \circ C_{x y w} \\
&=\varphi_{x y}^{-1}\left[K_{x y, \alpha} \circ H_{y z, \beta} \circ \varphi_{y z}^{-1}\left[K_{y z} \circ H_{z w, \gamma}\right]\right] \circ \varphi_{x y}^{-1}\left[K_{x y}{ }^{\circ} C_{y z w}\right] \circ C_{x y w},
\end{aligned}
$$

by (10), (14), the identity element properties of $K_{x y}$ with respect to its cosets, the fact that $K_{x y}$ is a normal subgroup of $G_{y}$ and therefore commutes with the other sets, and the isomorphism properties of $\varphi_{x y}^{-1}$. In this regard, observe that the complex product $K_{x y}{ }^{\circ} C_{y z w}$ is a union of cosets of $K_{x y}$ (this was the point of introducing $K_{x y}$ into the fourth expression), and of course so is

$$
K_{x y, \alpha} \circ H_{y z, \beta} \circ \varphi_{y z}^{-1}\left[K_{y z} \circ H_{z w, \gamma}\right]
$$

(since the coset $K_{x y, \alpha}$ is present in the complex product), so the isomorphism property of $\varphi_{x y}^{-1}$ for unions of cosets of $K_{x y}$ really is applicable.

This last string of equalities shows that (13) may be rewritten in the form

$$
\begin{aligned}
\bigcup\left\{R_{x w, \rho}:\right. & H_{x w, \rho} \subseteq \\
& \left.\varphi_{x y}^{-1}\left[K_{x y, \alpha} \circ H_{y z, \beta} \circ \varphi_{y z}^{-1}\left[K_{y z} \circ H_{z w, \gamma}\right]\right] \circ \varphi_{x y}^{-1}\left[K_{x y}{ }^{\circ} C_{y z w}\right] \circ C_{x y w}\right\},
\end{aligned}
$$

so (9) is equal to (15).

It has been shown that (11) is equal to (8), and (9) to (15). It follows that (1) and (9) will be equal, that is to say, condition (i) of the theorem will hold, just in case (8) and (15) are equal. According to Lemma 3.3, the unions (8) and (15) are equal just in case the corresponding cosets

$$
\varphi_{x y}^{-1}\left[K_{x y, \alpha}{ }^{\circ} H_{y z, \beta}{ }^{\circ} \varphi_{y z}^{-1}\left[K_{y z} \circ H_{z w, \gamma}\right]\right]{ }^{\circ} C_{x y z}{ }^{\circ} C_{x z w}
$$

and

$$
\varphi_{x y}^{-1}\left[K_{x y, \alpha}{ }^{\circ} H_{y z, \beta}{ }^{\circ} \varphi_{y z}^{-1}\left[K_{y z} \circ H_{z w, \gamma}\right]\right] \circ \varphi_{x y}^{-1}\left[K_{x y}{ }^{\circ} C_{y z w}\right] \circ C_{x y w}
$$


are equal. Apply the cancellation law for the quotient group $G_{x} /\left(H_{x y}{ }^{\circ} H_{x z} \circ H_{x w}\right)$ to conclude that these two cosets are equal if and only if

$$
C_{x y z}{ }^{\circ} C_{x z w}=\varphi_{x y}^{-1}\left[K_{x y}{ }^{\circ} C_{y z w}\right]{ }^{\circ} C_{x y w} .
$$

To justify this application of the cancellation law, it must be shown that the relevant factors, namely

$$
\begin{gathered}
\varphi_{x y}^{-1}\left[K_{x y, \alpha}{ }^{\circ} H_{y z, \beta}{ }^{\circ} \varphi_{y z}^{-1}\left[K_{y z} \circ H_{z w, \gamma}\right]\right], \\
C_{x y z}{ }^{\circ} C_{x z w},
\end{gathered}
$$

$$
\varphi_{x y}^{-1}\left[K_{x y}{ }^{\circ} C_{y z w}\right] \circ C_{x y w},
$$

really are all cosets in $G_{x}$ of the normal subgroup

$$
H_{x y} \circ H_{x z} \circ H_{x w} \text {. }
$$

Begin with (17). Observe that $K_{y z} \circ H_{z w, \gamma}$ is a coset of $K_{y z} \circ H_{z w}$, so its inverse image under $\varphi_{y z}$ is a coset of $H_{y z}{ }^{\circ} H_{y w}$. The complex product $K_{x y, \alpha}{ }^{\circ} H_{y z, \beta}$ is a coset of $K / H$, so the product

$$
K_{x y, \alpha} \circ H_{y z, \beta} \circ \varphi_{y z}^{-1}\left[K_{y z} \circ H_{z w, \gamma}\right]
$$

is a coset of the group $K_{x y} \circ H_{y z} \circ H_{y z} \circ H_{y w}$, which coincides with the group

$$
K_{x y} \circ H_{y z} \circ H_{y w} \text {. }
$$

Applying $\varphi_{x y}^{-1}$ to (21) gives (17). Applying it to (22) gives

$$
\varphi_{x y}^{-1}\left[K_{x y} \circ H_{y z} \circ H_{y w}\right] .
$$

Since

$$
\begin{aligned}
\varphi_{x y}^{-1}\left[K_{x y} \circ H_{y z} \circ H_{y w}\right] & =\varphi_{x y}^{-1}\left[K_{x y} \circ K_{x y} \circ H_{y z} \circ H_{y w}\right] \\
& =\varphi_{x y}^{-1}\left[K_{x y} \circ H_{y z} \circ K_{x y} \circ H_{y w}\right] \\
& =\varphi_{x y}^{-1}\left[K_{x y} \circ H_{y z} \circ^{\circ} \varphi_{x y}^{-1}\left[K_{x y} \circ H_{y w}\right]\right. \\
& =\left(H_{x y} \circ H_{x z}\right)^{\circ}\left(H_{x y} \circ H_{x w}\right) \\
& =H_{x y} \circ H_{x y} \circ H_{x z}{ }^{\circ} H_{x w} \\
& =H_{x y} \circ H_{x z} \circ H_{x w},
\end{aligned}
$$

and since (21) is a coset of (22), it may be concluded that (17) is a coset of (20), as claimed.

Turn now to (18). By assumption, $C_{x y z}$ is a coset of the subgroup $H_{x y}{ }^{\circ} H_{x z}$, and $C_{x z w}$ is a coset of the subgroup $H_{x y}{ }^{\circ} H_{x w}$, so the product coset (18) is a coset of the product subgroup, which is (20).

Consider, finally, (19). By assumption, $C_{y z w}$ is a coset of $H_{y z}{ }^{\circ} H_{y w}$, so the product $K_{x y}{ }^{\circ} C_{y z w}$ is a coset of $K_{x y}{ }^{\circ} H_{y z}{ }^{\circ} H_{y w}$. It follows that the inverse image

$$
\varphi_{x y}^{-1}\left[K_{x y}{ }^{\circ} C_{y z w}\right]
$$

is a coset of the inverse image $\varphi_{x y}^{-1}\left[K_{x y} \circ H_{y z} \circ H_{y w}\right]$. It was shown above that this inverse image coincides with (20), so (23) is a coset of (20). The set $C_{x w y}$ is a coset of $H_{x w}{ }^{\circ} H_{x y}$, by assumption, so the product of $C_{x w y}$ with (23) is a coset of the product of $H_{x w}{ }^{\circ} H_{x y}$ with (20). This last product reduces to (20), so (19)) is a coset of (20). 
We carry out one final transformation of (16). Semi-frame condition (ii) says that $\varphi_{y x}$ is the inverse of $\varphi_{x y}$, and consequently $K_{x y}$ coincides with the subgroup $H_{y x}$, by Convention 2.6. Also, the subgroup $H_{y x}$ is normal. Consequently, equation (16) may be rewritten in the form

$$
C_{x y z}{ }^{\circ} C_{x z w}=\varphi_{y x}\left[C_{y z w}{ }^{\circ} H_{y x}\right] \circ C_{x y w},
$$

which is just the equation in condition (iii).

It has been demonstrated that condition (i) holds for the fixed $\alpha, \beta$, and $\gamma$ just in case the equation in condition (iii) holds. Since the formulation of (iii) does not involve any of the three given indices, it follows that (iii) implies (i) for each such triple of indices, and therefore (iii) implies (ii). The implication from (ii) to (i) is obvious.

The associative law holds in $\mathfrak{C}[\mathcal{F}]$ just in case it holds for all atoms. Consider a triple of atoms

$$
R_{x y, \alpha}, \quad R_{w z, \beta}, \quad R_{u v, \gamma} .
$$

If $y=w$ and $z=u$, then the law holds for the triple of atoms just in case

$$
C_{x y z}{ }^{\circ} C_{x z w}=\varphi_{y x}\left[C_{y z w} \circ H_{y x}\right] \circ C_{x y w},
$$

by the equivalence of conditions (ii) and (iii) in the first part of the theorem.

If $y \neq w$ or if $z \neq u$, then the associative law holds automatically for this triple, since both sides reduce to the empty relation. Indeed, if $y \neq w$, then

$$
R_{x y, \alpha} \otimes R_{w z, \beta}=\varnothing,
$$

by the definition of $\otimes$, and consequently

$$
\left(R_{x y, \alpha} \otimes R_{w z, \beta}\right) \otimes R_{u v, \gamma}=\varnothing,
$$

again, by the definition of $\otimes$. If also $z \neq u$, then a similar argument shows that

$$
R_{x y, \alpha} \otimes\left(R_{w z, \beta} \otimes R_{u v, \gamma}\right)=\varnothing .
$$

In this case, associativity holds by (24) and (25).

If $z=u$, then the argument is slightly more involved. In this case,

$$
R_{w z, \beta} \otimes R_{u v, \gamma}=\bigcup\left\{R_{w v, \xi}: H_{w v, \xi} \subseteq \varphi_{w z}^{-1}\left[K_{w z, \beta} \circ H_{u v, \gamma}\right]{ }^{\circ} C_{w z v}\right\},
$$

by the definition of $\otimes$, and therefore

$$
\begin{aligned}
R_{x y, \alpha} \otimes\left(R_{w z, \beta} \otimes R_{u v, \gamma}\right) & = \\
\bigcup & \left\{R_{x y, \alpha} \otimes R_{w v, \xi}: H_{w v, \xi} \subseteq \varphi_{w z}^{-1}\left[K_{w z, \beta} \circ H_{u v, \gamma}\right] \circ C_{w z v}\right\},
\end{aligned}
$$

by (26) and the distributivity of the operation $\otimes$ over arbitrary unions. Each of the relations $R_{x y, \alpha} \otimes R_{w v, \xi}$ in this union is empty, by the definition of $\otimes$, since we have assumed that $y \neq w$. It follows that (25) holds in this case as well. Compare (25) with (24) to arrive at the desired conclusion for the case $y \neq w$. The case $z \neq u$ is treated in an analogous fashion.

The next corollary says that semi-frame condition (iv) is necessary for $\mathfrak{C}[\mathcal{F}]$ to be a relation algebra.

Corollary 3.9 (Semi-frame Corollary). Assume that $\mathcal{F}$ is a pre-semi-frame. If either the Second Involution Law or the Associative Law holds in the algebra $\mathfrak{C}[\mathcal{F}]$, then $\mathcal{F}$ is a semi-frame. 
Proof. Assume that the Second Involution Law holds in $\mathfrak{C}[\mathcal{F}]$. Semi-frame condition (iv) was used only once in the proof of Theorem 3.7 when Lemma 3.6(ii) was applied to justify the third equality in (13). Omitting that step, the proof shows that Theorem 3.7(i) holds just in case the cosets in (16) and the modified (17) of that proof are equal, that is to say, just in case

$$
C_{x y z}^{-1}{ }^{\circ} \varphi_{x y}^{-1}[Q]=\varphi_{x z}^{-1}\left[\varphi_{y z}[Q]\right] \circ \varphi_{z x}\left[C_{z y x}\right],
$$

where $Q$ is $H_{y z, \beta}^{-1}{ }^{\circ} K_{x y, \alpha}^{-1}$. From the assumption that the Second Involution Law holds, it follows that (11) holds for all $\alpha, \beta$, that is to say, for all cosets $Q$ of $H_{y z} \circ K_{x y}$. Take $Q=H_{y z} \circ K_{x y}$ and use semi-frame condition (iii) to obtain $\varphi_{x y}^{-1}[Q]=\varphi_{x z}^{-1}\left[\varphi_{y z}[Q]\right]$. Substitute the left side of this equality for the right side in (11), and use the cancellation law for groups (and the fact that for this choice of $Q$, the inverse image $\varphi_{x y}^{-1}(Q)$ is a normal subgroup of $G_{x}$, and hence commutes with $\left.\varphi_{z x}\left(C_{z y x}\right)\right)$ to reduce (11) to

$$
C_{x y z}^{-1}=\varphi_{z x}\left[C_{z y x}\right] .
$$

Substitute the left side of (2) for the right side in equation (1), and then multiply both sides of the resulting equation by $C_{x y z}$ on the right to arrive at Lemma 3.6(ii), which is equivalent to Lemma 3.6(i). Thus, Lemma 3.6(i) holds for all triples $(x, y, z)$ in $\mathcal{E}_{3}$, which is just what semi-frame condition (iv) expresses.

Assume now that the Associative Law holds in $\mathfrak{C}[\mathcal{F}]$. The derivation of semiframe condition (iv) is similar to the preceding one. Semi-frame condition (iv) was used only once in the proof of Theorem 3.8, when Lemma 3.6(iii) was applied to justify the sixth equality in the transformation of the expression $C_{x y z}{ }^{\circ} \varphi_{x z}^{-1}\left[K_{x z}{ }^{\circ} H_{z w, \gamma}\right]$. If we use the "half-transformed" expression that we get without using Lemma 3.6(iii), in place of the one in step (8) of that proof, we get the term

$$
\varphi_{x y}^{-1}\left[K_{x y, \alpha} \circ H_{y z, \beta}\right] \circ C_{x y z} \circ \varphi_{x z}^{-1}\left[K_{x z} \circ K_{y z} \circ H_{z w, \gamma}\right] \circ C_{x z w} .
$$

Theorem 3.8(i) is equivalent to the equality of (3) and the term in (15) of that proof, that is to say, to the term

$$
\varphi_{x y}^{-1}\left[K_{x y, \alpha} \circ H_{y z, \beta}{ }^{\circ} \varphi_{y z}^{-1}\left[K_{y z} \circ H_{z w, \gamma}\right]\right]{ }^{\circ} \varphi_{x y}^{-1}\left[K_{x y}{ }^{\circ} C_{y z w}\right] \circ C_{x y w} .
$$

Multiply the two terms on the left by $\varphi_{x y}\left[K_{x y, \alpha} \circ H_{y z, \beta}\right]$, use isomorphism property of $\varphi_{x y}^{-1}$, and write $Q$ in place of $K_{y z}{ }^{\circ} H_{z w, \gamma}$ to get that Theorem $3.8(\mathrm{i})$ is equivalent to the equation

$$
C_{x y z}{ }^{\circ} \varphi_{x z}^{-1}\left[K_{x z} \circ Q\right] \circ C_{x z w}=\varphi_{x y}^{-1}\left[\varphi_{y z}^{-1}[Q]\right] \circ \varphi_{x y}^{-1}\left[K_{x y}{ }^{\circ} C_{y z w}\right] \circ C_{x y w} .
$$

The assumption that the associative law holds implies that (5) holds for all cosets $Q$ of $K_{y z}{ }^{\circ} H_{z w}$. In particular, it holds for $K_{y z}{ }^{\circ} H_{z w}$, from which it follows that

$$
C_{x y z}{ }^{\circ} C_{x z w}=\varphi_{x y}^{-1}\left[K_{x y} \circ C_{y z w}\right] \circ C_{x y w} .
$$

Substitute the left side of (6) for the right side in its occurrence on the right side of (5), and then cancel the occurrence of $C_{x z w}$ on the right of both sides of the resulting equation, to get Lemma 3.6(iii). The desired conclusion now follows just as in the previous paragraph.

Coset relation algebras are generalizations of group relation algebras, since each group relation algebra may be viewed as a coset relation algebra. In more detail, let $\mathcal{F}=(G, \varphi)$ be a group frame, and put $\overline{\mathcal{F}}=(G, \varphi, C)$ where $C_{x y z}=H_{x y} \circ H_{x z}$ for each triple $(x, y, z)$ in $\mathcal{E}_{3}$. It is easy to see that the algebras $\mathfrak{G}[\mathcal{F}]$ and $\mathfrak{C}[\overline{\mathcal{F}}]$ are 
equal. In Section 5 it will be shown the class of coset relation algebras is a proper extension of the class of group relation algebras: there exist coset relation algebras that are not group relation algebras.

We conclude the present section with two lemmas that concern the relationship between these two constructions. The first lemma characterizes when the operation $\otimes$ gives the same result as relational composition.

Lemma 3.10. Let $\mathcal{F}$ be a semi-frame. The following conditions are equivalent for all triples $(x, y, z)$ in $\mathcal{E}_{3}$.

(i) $R_{x y, \alpha} \otimes R_{y z, \beta}=R_{x y, \alpha} \mid R_{y z, \beta}$ for some $\alpha<\kappa_{x y}$ and some $\beta<\kappa_{y z}$.

(ii) $R_{x y, \alpha} \otimes R_{y z, \beta}=R_{x y, \alpha} \mid R_{y z, \beta}$ for all $\alpha<\kappa_{x y}$ and all $\beta<\kappa_{y z}$.

(iii) $C_{x y z}=H_{x y}{ }^{\circ} H_{x z}$.

Proof. Assume first that condition (iii) holds, with the goal of establishing (ii). Clearly,

$$
\varphi_{x y}^{-1}\left[K_{x y, \alpha}{ }^{\circ} H_{y z, \beta}\right]{ }^{\circ} C_{x y z}=\varphi_{x y}^{-1}\left[K_{x y, \alpha}{ }^{\circ} H_{y z, \beta}\right],
$$

because $H_{x y} \circ H_{x z}$ is the identity element in its group of cosets. For the same reason, the inner automorphism $\tau$ of $G_{x} /\left(H_{x y}{ }^{\circ} H_{x z}\right)$ determined by the coset $C_{x y z}$ is the identity automorphism. Semi-frame condition (iv) therefore reduces to

$$
\hat{\varphi}_{x y}\left|\hat{\varphi}_{y z}=\tau\right| \hat{\varphi}_{x z}=\hat{\varphi}_{x z} .
$$

Use (2) and the implication from (iv) to (iii) in the Composition Theorem to obtain

$$
R_{x y, \alpha} \mid R_{y z, \beta}=\bigcup\left\{R_{x z, \gamma}: H_{x z, \gamma} \subseteq \varphi_{x y}^{-1}\left[K_{x y, \alpha}{ }^{\circ} H_{y z, \beta}\right]\right\}
$$

for all $\alpha<\kappa_{x y}$ and $\beta<\kappa_{y z}$. (The first hypothesis in condition (iv) is satisfied because of semi-frame condition (iii).) Use Definition 3.1 to get

$$
R_{x y, \alpha} \otimes R_{y z, \beta}=\bigcup\left\{R_{x z, \gamma}: H_{x z, \gamma} \subseteq \varphi_{x y}^{-1}\left[K_{x y, \alpha} \circ H_{y z, \beta}\right] \circ C_{x y z}\right\}
$$

for all $\alpha<\kappa_{x y}$ and all $\beta<\kappa_{y z}$. Combine (3), (4), and (11) to arrive at (ii).

The implication from (ii) to (i) is obvious. To establish the implication from (i) to (iii), let $\alpha<\kappa_{x y}$ and $\beta<\kappa_{y z}$ be fixed indices such that (i) holds. Since the universe $A$ of the algebra $\mathfrak{C}[\mathcal{F}]$ is closed under the operation $\otimes$, the composition $R_{x y, \alpha} \mid R_{y z, \beta}$ must belong to $A$. Apply Corollary 2.9 to see that this composition must belong to $A$ for every choice of $\alpha<\kappa_{x y}$ and $\beta<\kappa_{y z}$. Invoke the Composition Theorem to obtain (3). Use Definition 3.1 to get (44). Combine (3) and (4) with the assumption in (i) to arrive at

$$
\begin{aligned}
\bigcup\left\{R_{x z, \gamma}: H_{x z, \gamma}\right. & \left.\subseteq \varphi_{x y}^{-1}\left[K_{x y, \alpha}{ }^{\circ} H_{y z, \beta}\right]\right\} \\
& =\bigcup\left\{R_{x z, \gamma}: H_{x z, \gamma} \subseteq \varphi_{x y}^{-1}\left[K_{x y, \alpha} \circ H_{y z, \beta}\right]{ }^{\circ} C_{x y z}\right\}
\end{aligned}
$$

for the $\alpha$ and $\beta$ chosen so that (i) holds. Apply Lemma 3.3 to (5) to obtain (11). (To check that Lemma 3.3 really is applicable, observe that the inverse image $\varphi_{x y}^{-1}\left[K_{x y, \alpha}{ }^{\circ} H_{y z, \beta}\right]$ of the coset $K_{x y, \alpha}{ }^{\circ} H_{y z, \beta}$ of $K / H$ is a coset of $H_{x y}{ }^{\circ} H_{x z}$, because $\hat{\varphi}_{x y}$ maps $G_{x} /\left(H_{x y}{ }^{\circ} H_{x z}\right)$ isomorphically to $G_{y} /(K / H)$. Also, $C_{x y z}$ is a coset of $H_{x y}{ }^{\circ} H_{x z}$, by assumption. Consequently, the composition

$$
\varphi_{x y}^{-1}\left[K_{x y, \alpha}{ }^{\circ} H_{y z, \beta}\right] \circ C_{x y z}
$$

is a coset of $H_{x y}{ }^{\circ} H_{x z}$.) The only element of a (quotient) group that leaves another element of the group unchanged under group composition is the identity element, 
by the cancellation law for groups. Consequently, it follows from (11) that $C_{x y z}$ must coincide with the identity element $H_{x y}{ }^{\circ} H_{x z}$ of the quotient group.

In general, the composition $R_{x y, \alpha} \mid R_{y z, \beta}$ does not belong to the algebra $\mathfrak{C}[\mathcal{F}]$. Fortunately, it is possible to characterize when it does belong.

Lemma 3.11. Let $\mathcal{F}$ be a semi-frame. The following conditions are equivalent for all triples $(x, y, z)$ in $\mathcal{E}_{3}$.

(i) $R_{x y, \alpha} \mid R_{y z, \beta}$ is in $\mathfrak{C}[\mathcal{F}]$ for some $\alpha<\kappa_{x y}$ and some $\beta<\kappa_{y z}$.

(ii) $R_{x y, \alpha} \mid R_{y z, \beta}$ is in $\mathfrak{C}[\mathcal{F}]$ for all $\alpha<\kappa_{x y}$ and all $\beta<\kappa_{y z}$.

(iii) $C_{x y z}$ is in the center of the group $G_{x} /\left(H_{x y} \circ H_{x z}\right)$.

Proof. The equivalence of (i) and (ii) is proved in Corollary 2.9. To establish the implication from (iii) to (ii), assume that $C_{x y z}$ is in the center of $G_{x} /\left(H_{x y} \circ H_{x z}\right)$. The inner automorphism $\tau$ determined by $C_{x y z}$ is then the identity automorphism, so semi-frame condition (iv) for the given triple $(x, y, z)$ reduces to

$$
\hat{\varphi}_{x y} \mid \hat{\varphi}_{y z}=\hat{\varphi}_{x z} .
$$

Keeping in mind semi-frame condition (iii), we see that the conditions in part (iv) of the Composition Theorem are satisfied for the triple $(x, y, z)$. By the implication from (iv) to (ii) in that theorem, the composition $R_{x y, \alpha} \mid R_{y z, \beta}$ must be in the universe $A$ of the algebra $\mathfrak{C}[\mathcal{F}]$ for all $\alpha$ and $\beta$.

To establish the implication from (ii) to (iii), assume that $R_{x y, \alpha} \mid R_{y z, \beta}$ is in $A$ for all $\alpha$ and $\beta$. It follows from the Composition Theorem that (11) holds. By assumption, $\mathcal{F}$ is a semi-frame, so

$$
\hat{\varphi}_{x y}\left|\hat{\varphi}_{y z}=\tau\right| \hat{\varphi}_{x z},
$$

with $\tau$ denoting $\tau_{x y z}$. Comparing (11) and (2), it is clear that

$$
\hat{\varphi}_{x z}=\tau \mid \hat{\varphi}_{x z} \text {. }
$$

Form the relational composition of each side of this equation with $\hat{\varphi}_{x z}^{-1}$ on the right to see that $\tau$ is the identity automorphism of the quotient group $G_{x} /\left(H_{x y} \circ H_{x z}\right)$. This can only happen if $C_{x y z}$ is in the center of the quotient group, because $\tau$ is the inner automorphism determined by $C_{x y z}$.

\section{Coset Semi-Frames}

In the preceding section, necessary and sufficient conditions are given for the algebra $\mathfrak{C}[\mathcal{F}]$ constructed from a coset semi-frame $\mathcal{F}$ to satisfy the identity law, the second involution law, the cycle law, and the associative law, and hence to be a relation algebra. We single out the coset semi-frames that satisfy these conditions.

Definition 4.1. A coset semi-frame

$$
\mathcal{F}=\left(\left\langle G_{x}: x \in I\right\rangle,\left\langle\varphi_{x y}:(x, y) \in \mathcal{E}\right\rangle,\left\langle C_{x y z}:(x, y, z) \in \mathcal{E}_{3}\right\rangle\right)
$$

is said to satisfy the coset conditions if the following equations hold for all pairs $(x, y)$ in $\mathcal{E}$, all triples $(x, y, z)$ in $\mathcal{E}_{3}$, and all quadruples $(x, y, z, w)$ in $\mathcal{E}_{4}$ respectively.

(i) $C_{x y y}=H_{x y}$.

(ii) $\varphi_{x z}\left[C_{x y z}\right]=C_{z y x}^{-1}$.

(iii) $\varphi_{x y}\left[C_{x y z}\right]=C_{y x z}^{-1}$.

(iv) $C_{x y z}{ }^{\circ} C_{x z w}=\varphi_{y x}\left[C_{y z w}{ }^{\circ} H_{y x}\right] \circ C_{x y w}$. 
These are called the coset conditions for the identity law, the second involution law, the cycle law, and the associative law respectively.

The results in the previous section lead to the following theorem, which is one of the main results of this paper.

Theorem 4.2 (Coset Semi-frame Theorem). If a coset semi-frame $\mathcal{F}$ satisfies the coset conditions, then the algebra $\mathfrak{C}[\mathcal{F}]$ constructed from $\mathcal{F}$ is a complete and atomic measurable relation algebra with base set and unit

$$
U=\bigcup\left\{G_{x}: x \in I\right\} \quad \text { and } \quad E=\bigcup\left\{G_{x} \times G_{y}:(x, y) \in \mathcal{E}\right\}
$$

respectively. The atoms in this algebra are the relations of the form $R_{x y, \alpha}$ for pairs $(x, y)$ in $\mathcal{E}$, and the subidentity atoms are the relations of the form $R_{x x, 0}$ for elements $x$ in $I$. The measure of $R_{x x, 0}$ is just the cardinality of the group $G_{x}$.

Proof. The algebra $\mathfrak{C}[\mathcal{F}]$ is a complete and atomic Boolean algebra of binary relations containing the identity relation $i d_{U}$, and closed under the set-theoretic operation of converse and under the operation $\otimes$, by the definition of a semi-frame, the assumption that $\mathcal{F}$ is a semi-frame, and Boolean Algebra Theorem 2.3. Identity Theorem 2.4, Converse Theorem 2.5, and the definition of $\otimes$. The Boolean axioms (R1)-(R3), the first involution law (R6), and the two distributive laws (R8) and (R9) are valid in $\mathfrak{C}[\mathcal{F}]$, by Theorem 2.3 and the remarks following Definition 3.2. The associative law (R4), the identity law (R5), the second involution law (R7), and the cycle law (R11) are also valid in $\mathfrak{C}[\mathcal{F}]$, by Associative Law Theorem 3.8. Identity Law Theorem 3.4. Second Involution Law Theorem 3.7 and Cycle Law Theorem 3.5 respectively, because $\mathcal{F}$ is assumed to satisfy the coset conditions. Consequently, $\mathfrak{C}[\mathcal{F}]$ is a complete and atomic relation algebra in which the universe consists of binary relations, and all operations except the one for relative multiplication, coincide with the standard set-theoretic operations of set relation algebras.

The atoms of the algebra $\mathfrak{C}[\mathcal{F}]$ are the relations of the form $R_{x y, \alpha}$, and the subidentity atoms are the relations of the form $R_{x x, 0}$, by Lemma 2.2, Theorem 2.3 . and the construction of $\mathfrak{C}[\mathcal{F}]$. The identity relation $i d_{U}$ is the disjoint union of the subidentity atoms $R_{x x, 0}$, by Theorem 2.4 and semi-frame condition (i).

To prove that each subidentity atom $R_{x x, 0}$ is measurable, with measure the cardinality of the group $G_{x}$, it must be shown that the square

$$
R_{x x, 0} \otimes E \otimes R_{x x, 0}
$$

is a union of $\kappa_{x x}$ non-zero functional atoms. The unit $E$ may be written in the form

$$
E=\bigcup\left\{G_{y} \times G_{z}:(y, z) \in \mathcal{E}\right\}=\bigcup\left\{R_{y z, \alpha}:(y, z) \in \mathcal{E} \text { and } \alpha<\kappa_{y z}\right\},
$$

by Lemma 2.2 and Theorem 2.3. Consequently,

$$
\begin{aligned}
R_{x x, 0} \otimes E \otimes R_{x x, 0} & =R_{x x, 0} \otimes\left(\bigcup\left\{R_{y z, \alpha}:(y, z) \in \mathcal{E} \text { and } \alpha<\kappa_{y z}\right\}\right) \otimes R_{x x, 0} \\
& =\bigcup\left\{R_{x x, 0} \otimes R_{y z, \alpha} \otimes R_{x x, 0}:(y, z) \in \mathcal{E} \text { and } \alpha<\kappa_{y z}\right\},
\end{aligned}
$$

by (2) and the distributivity of $\otimes$ over arbitrary unions. If $x \neq y$ or $x \neq z$, then

$$
R_{x x, 0} \otimes R_{y z, \alpha} \otimes R_{x x, 0}=\varnothing,
$$


by the definition of the operation $\otimes$. On the other hand, if $x=y$ and $x=z$, then

$$
\begin{aligned}
R_{x x, 0} \otimes R_{y z, \alpha} \otimes R_{x x, 0}=R_{x x, 0} \otimes R_{x x, \alpha} \otimes & R_{x x, 0} \\
& =R_{x x, 0}\left|R_{x x, \alpha}\right| R_{x x, 0}=R_{x x, \alpha} .
\end{aligned}
$$

The first equality uses the assumptions on $y$ and $z$. The second equality uses the assumption that $\mathcal{F}$ satisfies the coset condition for the identity law, together with Lemma 3.10 and Theorem 3.4, which ensures that condition (iii) of Lemma 3.10. namely

$$
C_{x y z}=C_{x x x}=H_{x x}=H_{x x} \circ H_{x x}=H_{x y} \circ H_{x z},
$$

is satisfied. The third equality uses the fact that $R_{x x, 0}=i d_{G_{x}}$, and $R_{x x, \alpha}$ is a subset of $G_{x} \times G_{x}$. Combine (3) -(5), and use Lemma 2.2 to arrive at

$$
R_{x x, 0} \otimes E \otimes R_{x x, 0}=\bigcup\left\{R_{x x, \alpha}: \alpha<\kappa_{x x}\right\}=G_{x} \times G_{x} .
$$

Since $H_{x x}=K_{x x}=\left\{e_{x}\right\}$, the sets $H_{x x, \gamma}=K_{x x, \gamma}$ have the form $\left\{g_{\gamma}\right\}$, and therefore the relations $R_{x x, \alpha}$ (for $\alpha<\kappa_{x x}$ ) have the form

$$
\begin{aligned}
R_{x x, \alpha}=\bigcup_{\gamma}\left\{H_{x x, \gamma} \times\right. & \left.\left(K_{x x, \gamma} \circ K_{x x, \alpha}\right)\right\} \\
& =\bigcup_{\gamma}\left\{\left\{g_{\gamma}\right\} \times\left\{g_{\gamma} \circ g_{\alpha}\right\}\right\}=\left\{\left(g_{\gamma}, g_{\gamma} \circ g_{\alpha}\right): \gamma<\kappa_{x x}\right\},
\end{aligned}
$$

which is a function, and in fact a bijection.

It follows from (7) and (8) that the square (11) is the disjoint union of $\kappa_{x x}$ functions. Consequently, $R_{x x, 0}$ is a measurable atom of measure $\kappa_{x x}$. Combine this with the observations of the previous paragraph to conclude that the relation algebra $\mathfrak{C}[\mathcal{F}]$ is measurable.

The theorem justifies the following definition.

Definition 4.3. Suppose that $\mathcal{F}$ is a coset semi-frame that satisfies the coset conditions. The relation algebra $\mathfrak{C}[\mathcal{F}]$ constructed from $\mathcal{F}$ in Coset Semi-frame Theorem 4.2 is called the (full) coset relation algebra on $\mathcal{F}$. A general coset relation algebra is defined to be an algebra that is embeddable into a full coset relation algebra.

The task of verifying that a given group triple satisfies the semi-frame conditions and the coset conditions, and therefore yields a full coset relation algebra, that is to say, it yields an example of a measurable relation algebra, can be quite complicated and tedious. Fortunately, some simplifications are possible. To describe them, it is helpful to assume that the group index set $I$ is linearly ordered, say by a relation $<$. Roughly speaking, under the assumption of condition (i), condition (ii) holds in general just in case it holds for each pair $(x, y)$ in $\mathcal{E}$ with $x<y$, and similarly for the other semi-frame conditions. Similar simplifications are possible for most of the remaining semi-frame and coset conditions. Actually, it is possible to replace coset conditions (i) - (iii) with four simpler conditions that do not simultaneously involve the formation of a coset inverse and the application of a quotient isomorphism.

We begin with two lemmas. The first formulates some conditions that are equivalent to coset condition (ii) for the second involution law and coset condition (iii) for the cycle law.

Lemma 4.4. Let $\mathcal{F}$ be a semi-frame, and $(u, v, w)$ a triple in $\mathcal{E}_{3}$. Consider the following conditions on the coset system of $\mathcal{F}$. 
(i) $C_{x y z}^{-1}=C_{x z y}$ for all permutations $(x, y, z)$ of $(u, v, w)$.

(ii) $\varphi_{x z}\left[C_{x y z}\right]=C_{z y x}^{-1} \quad$ for all permutations $(x, y, z)$ of $(u, v, w)$.

(iii) $\varphi_{x z}\left[C_{x y z}\right]=C_{z x y}$ for all permutations $(x, y, z)$ of $(u, v, w)$.

(iv) $\varphi_{x y}\left[C_{x y z}\right]=C_{y x z}^{-1}$ for all permutations $(x, y, z)$ of $(u, v, w)$.

(v) $\varphi_{x y}\left[C_{x y z}\right]=C_{y z x}$ for all permutations $(x, y, z)$ of $(u, v, w)$.

Conditions (iii) and (v) are equivalent. Any two of conditions (i)-(iv), and also any two of conditions (i), (ii), (iv) and (v), imply all of the other conditions.

Proof. First, observe that

$$
\varphi_{y z}\left[\varphi_{x y}\left[C_{x y z}\right]\right]=\varphi_{x z}\left[C_{x y z}\right]
$$

holds by semi-frame condition (iv), since

$$
\tau_{x y z}\left(C_{x y z}\right)=C_{x y z}^{-1}{ }^{\circ} C_{x y z} \circ C_{x y z}=C_{x y z} .
$$

Apply $\varphi_{z y}$ to both sides of (1), and use the fact that $\varphi_{z y}$ is the inverse of $\varphi_{y z}$, by semi-frame condition (ii), to obtain

$$
\varphi_{z y}\left[\varphi_{x z}\left[C_{x y z}\right]\right]=\varphi_{x y}\left[C_{x y z}\right] .
$$

The equivalence of (iii) and (v) is now easy to prove. If (iii) holds, then

$$
C_{y z x}=\varphi_{z y}\left[C_{z x y}\right]=\varphi_{z y}\left[\varphi_{x z}\left[C_{x y z}\right]\right]=\varphi_{x y}\left[C_{x y z}\right],
$$

by (iii) (with $z, x$, and $y$ in place of $x, y$, and $z$ respectively), another application of (iii), and (2). On the other hand, if (v) holds, then

$$
C_{z x y}=\varphi_{y z}\left[C_{y z x}\right]=\varphi_{y z}\left[\varphi_{x y}\left[C_{x y z}\right]\right]=\varphi_{x z}\left[C_{x y z}\right],
$$

by (v) (with $y, z$, and $x$ in place of $x, y$, and $z$ respectively), another application of (v), and (1).

The next step is to show that conditions (i) and (ii) imply all of the remaining conditions. The derivation of (iii) and (iv) from (i) and (ii) is easy. For (iii), use (ii) and (i) (with $x$ and $z$ interchanged):

$$
\varphi_{x z}\left[C_{x y z}\right]=C_{z y x}^{-1}=C_{z x y} .
$$

For (iv), first use (i), (ii) (with $y$ and $z$ interchanged), and (i) (with $y, z$, and $x$ in place of $x, y$, and $z$ respectively) to get

$$
\varphi_{x y}\left[C_{x y z}^{-1}\right]=\varphi_{x y}\left[C_{x z y}\right]=C_{y z x}^{-1}=C_{y x z} .
$$

Form the coset inverses of the first and last terms, and use the isomorphism properties of $\varphi_{x y}$, to arrive at (iv). It has already been shown that (v) follows from (iii), so conditions (i) and (ii) do imply all of the remaining conditions.

To show that conditions (i) and (iii) imply all of the remaining conditions, it suffices to derive (ii), by the observations of the preceding paragraph. Use (iii) and (i) (with $x$ and $z$ interchanged) to obtain

$$
\varphi_{x z}\left[C_{x y z}\right]=C_{z x y}=C_{z y x}^{-1} .
$$

Similarly, to show that conditions (i) and (iv) imply all of the remaining conditions, it suffices to derive (ii). First, use (i), (iv) (with $y$ and $z$ interchanged), and (i) (with $z, x$, and $y$ in place of $x, y$, and $z$ respectively) to obtain

$$
\varphi_{x z}\left[C_{x y z}^{-1}\right]=\varphi_{x z}\left[C_{x z y}\right]=C_{z x y}^{-1}=C_{z y x} .
$$

Form the coset inverses of the first and last terms, and use the isomorphism properties of $\varphi_{x z}$, to arrive (ii). 
To prove that (ii) and (iii) imply all of the remaining conditions, it suffices to derive (i). Use (iii) and (ii) to get

$$
C_{z x y}=\varphi_{x z}\left[C_{x y z}\right]=C_{z y x}^{-1} .
$$

Interchange $x$ and $z$ to arrive at (i). Similarly, to prove that (ii) and (iv) imply all of the remaining conditions, it suffices to derive (i). Use (ii) (with $x$ and $y$ interchanged) and the isomorphism properties of $\varphi_{x y}$, (iv), (11), and (ii) to obtain

$$
C_{z x y}=\varphi_{y z}\left[C_{y x z}^{-1}\right]=\varphi_{y z}\left[\varphi_{x y}\left[C_{x y z}\right]\right]=\varphi_{x z}\left[C_{x y z}\right]=C_{z y x}^{-1} .
$$

Again, interchange $x$ and $z$ to arrive at (i).

Finally, to show that (iii) and (iv) imply the remaining conditions, it suffices to derive (i). Use (iii) (with $x$ and $y$ interchanged) and the isomorphism properties of $\varphi_{y z}$, (iv), (11), and (iii) to obtain

$$
C_{z y x}^{-1}=\varphi_{y z}\left[C_{y x z}^{-1}\right]=\varphi_{y z}\left[\varphi_{x y}\left[C_{x y z}\right]\right]=\varphi_{x z}\left[C_{x y z}\right]=C_{z x y} .
$$

As before, interchange $x$ and $z$ to arrive at (i).

The second lemma facilitates the verification of the second and third coset conditions in cases when some of the indices coincide.

Lemma 4.5. Let $\mathcal{F}$ be a semi-frame. If $C_{x y z}=H_{x y}{ }^{\circ} H_{x z}$ for every permutation $(x, y, z)$ of a given triple in $\mathcal{E}_{3}$, then

$$
C_{x y z}^{-1}=C_{x z y} \quad \text { and } \quad \varphi_{x y}\left[C_{x y z}\right]=C_{y z x}
$$

for every permutation of the given triple.

Proof. Assume that

$$
C_{x y z}=H_{x y} \circ H_{x z}
$$

for all permutations $(x, y, z)$ of a given triple in $\mathcal{E}_{3}$. Obviously,

$$
C_{x y z}^{-1}=\left(H_{x y} \circ H_{x z}\right)^{-1}=H_{x z}^{-1} \circ H_{x y}^{-1}=H_{x z} \circ H_{x y}=C_{x z y}
$$

for all such permutations, by (1), the second involution law for cosets, the fact that $H_{x z}$ and $H_{x y}$ are subgroups of $G_{x}$ and hence closed under inverses, and (1) (with $y$ and $z$ interchanged). Thus, the first equation in the conclusion holds.

Semi-frame conditions (ii) and (iii), together with Convention 2.6 and the fact that $H_{y x} \circ H_{y z}$ is a subgroup of $G_{y}$, imply that

$$
\varphi_{x y}\left[H_{x y} \circ H_{x z}\right]=K / H=H_{y x} \circ H_{y z}=\left(H_{y x} \circ H_{y z}\right)^{-1} .
$$

Consequently,

$$
\varphi_{x y}\left[C_{x y z}\right]=\varphi_{x y}\left[H_{x y} \circ H_{x z}\right]=\left(H_{y x} \circ H_{y z}\right)^{-1}=C_{y x z}^{-1}=C_{y z x},
$$

by (11), (2), (1) (with $x$ and $y$ interchanged), and the first conclusion of the lemma (with $x$ and $y$ interchanged). Thus, the second equation in the conclusion holds.

The next theorem formulates a set of simplified semi-frame and coset conditions.

Theorem 4.6. A group triple $\mathcal{F}$ is a coset semi-frame that satisfies the first three coset conditions if and only if the following eight conditions are satisfied.

(i) $\varphi_{x x}$ is the identity automorphism of $G_{x} /\left\{e_{x}\right\}$ for every $x$ in $I$.

(ii) $\varphi_{y x}=\varphi_{x y}^{-1}$ for every pair $(x, y)$ in $\mathcal{E}$ with $x<y$.

(iii) $\varphi_{x y}\left[H_{x y} \circ H_{x z}\right]=K_{x y} \circ H_{y z}$ and $\varphi_{y z}\left[K_{x y} \circ H_{y z}\right]=K_{x z} \circ K_{y z}$ for every triple $(x, y, z)$ in $\mathcal{E}_{3}$ with $x<y<z$. 
(iv) $\hat{\varphi}_{x y}\left|\hat{\varphi}_{y z}=\tau_{x y z}\right| \hat{\varphi}_{x z}$ for every triple $(x, y, z)$ in $\mathcal{E}_{3}$ with $x<y<z$.

(v) $C_{x x y}=C_{x y x}=C_{x y y}=H_{x y}$ for all pairs $(x, y)$ in $\mathcal{E}$.

(vi) $C_{x y z}^{-1}=C_{x z y}$ for all triples $(x, y, z)$ in $\mathcal{E}_{3}$ with $x, y, z$ mutually distinct.

(vii) $\varphi_{x y}\left[C_{x y z}\right]=C_{y z x}$ for all triples $(x, y, z)$ in $\mathcal{E}_{3}$ with $x<y<z$.

(viii) $\varphi_{x z}\left[C_{x y z}\right]=C_{z x y}$ for all triples $(x, y, z)$ in $\mathcal{E}_{3}$ with $x<y<z$.

If $\mathcal{F}$ is a group triple that satisfies conditions (i)-(viii), then $\mathcal{F}$ satisfies the fourth coset condition if and only if

(ix) $C_{x y z}{ }^{\circ} C_{x z w}=\varphi_{y x}\left[C_{y z w}{ }^{\circ} H_{y x}\right] \circ C_{x y w}$ for all quadruples $(x, y, z, w)$ in $\mathcal{E}_{4}$ with $x<y<z<w$.

Proof. Suppose that a group triple $\mathcal{F}$ satisfies conditions (i)-(viii) of the theorem. The proof that semi-frame conditions (i)-(iii) must hold is easy, and is in fact exactly the same as in the case of the corresponding simplification of the group frame conditions for group pairs (see Theorem 4.4 and its proof in [4). The details are therefore omitted. Turn to the verification of semi-frame condition (iv).

Consider a triple $(x, y, z)$ in $\mathcal{E}_{3}$, and assume first that not all of the indices are distinct, say $x=y$. The mapping $\varphi_{x y}$ is the identity automorphism of $G_{x} /\left\{e_{x}\right\}$, by condition (i), so that

$$
H_{x y}=H_{x x}=\left\{e_{x}\right\}=K_{x x}=K_{x y}, \quad H_{x z}=H_{y z}, \quad K_{x z}=K_{y z},
$$

and therefore

$$
H_{x y} \circ H_{x z}=H_{x z}, \quad K_{x y} \circ H_{y z}=H_{y z}=H_{x z}, \quad K_{x z} \circ K_{y z}=K_{y z} \circ K_{y z}=K_{y z} .
$$

It follows that the isomorphism $\hat{\varphi}_{x y}$ induced by $\varphi_{x y}$ on $G_{x} /\left(H_{x y}{ }^{\circ} H_{x z}\right)$ coincides with the identity automorphism of $G_{x} / H_{x z}$, the isomorphism $\hat{\varphi}_{y z}$ on $G_{y} /\left(K_{x y} \circ H_{y z}\right)$ coincides with $\varphi_{y z}$, and the isomorphism $\hat{\varphi}_{x z}$ induced by $\varphi_{x z}$ on $G_{x} /\left(H_{x y} \circ H_{x z}\right)$ coincides with $\varphi_{x z}$. On the other hand, the coset that determines the inner automorphism $\tau_{x y z}$ is the subgroup

$$
C_{x y z}=C_{x x z}=H_{x z}
$$

by condition (v), so that $\tau_{x y z}$ must be the identity automorphism of $G_{x} / H_{x z}$. Consequently,

$$
\hat{\varphi}_{x y}\left|\hat{\varphi}_{y z}=\varphi_{y z}=\varphi_{x z}=\tau_{x y z}\right| \hat{\varphi}_{x z}
$$

so semi-frame condition (iv) holds in this case. The cases when $y=z$ and when $x=z$ are treated in a completely analogous fashion.

It remains to consider the case when $x, y$, and $z$ are all distinct. Condition (vi) of the theorem implies that

$$
C_{x y z}^{-1}=C_{x z y}, \quad C_{y x z}^{-1}=C_{y z x}, \quad C_{z x y}^{-1}=C_{z y x},
$$

from which it follows that

$$
\tau_{x y z}^{-1}=\tau_{x z y}, \quad \tau_{y z x}^{-1}=\tau_{y x z}, \quad \tau_{z x y}^{-1}=\tau_{z y x} .
$$

For example, for every coset $D$ in $G_{y} /\left(H_{y z} \circ H_{y x}\right)$, we have

$$
\begin{aligned}
\tau_{y x z}\left(\tau_{y z x}(D)\right)=\tau_{y x z}\left(C_{y z x}^{-1} \circ D \circ C_{y z x}\right)= & C_{y x z}^{-1} \circ\left(C_{y z x}^{-1} \circ D \circ C_{y z x}\right) \circ C_{y x z} \\
& =C_{y z x}{ }^{\circ} C_{y z x}^{-1} \circ D \circ C_{y z x}{ }^{\circ} C_{y z x}^{-1}=D,
\end{aligned}
$$

by the definition of $\tau_{y z x}$, the definition of $\tau_{y x z}$, the second equation in (10), and the laws of group theory. This argument shows that the composition of $\tau_{y z x}$ and 
$\tau_{y x z}$ is the identity function on its domain. The same is also true of the reverse composition, so these two inner automorphisms are the inverses of one another.

The next step is to check that

$$
\tau_{x y z}\left|\hat{\varphi}_{x y}=\hat{\varphi}_{x y}\right| \tau_{y z x} \quad \text { and } \quad \tau_{x y z}\left|\hat{\varphi}_{x z}=\hat{\varphi}_{x z}\right| \tau_{z x y} .
$$

To verify the first equation, consider an arbitrary coset $D$ in $G_{x} /\left(H_{x y} \circ H_{x z}\right)$. The definition of $\tau_{x y z}$, the isomorphism properties of $\varphi_{x y}$, condition (vii) of the theorem, and the definition of $\tau_{y z x}$ imply that

$$
\begin{aligned}
\varphi_{x y}\left[\tau_{x y z}[D]\right]=\varphi_{x y}\left[C_{x y z}^{-1} \circ D \circ C_{x y z}\right]=\varphi_{x y}\left[C_{x y z}\right]^{-1} \circ \varphi_{x y}[D] \circ \varphi_{x y}\left[C_{x y z}\right] \\
=C_{y z x}^{-1} \circ \varphi_{x y}[D] \circ C_{y z x}=\tau_{y z x}\left[\varphi_{x y}[D]\right] .
\end{aligned}
$$

An analogous argument, using condition (viii) in place of condition (vii), establishes the second equation in (3).

Consider finally the case when all of the indices $x, y$, and $z$ are distinct. Assume $x<y<z$, and use condition (iv) of the theorem to obtain

$$
\hat{\varphi}_{x y}\left|\hat{\varphi}_{y z}=\tau_{x y z}\right| \hat{\varphi}_{x z} .
$$

Compose both sides of this equation on the right with $\hat{\varphi}_{y z}^{-1}$, and on the left with $\tau_{x y z}^{-1}$, to arrive at

$$
\tau_{x y z}^{-1}\left|\hat{\varphi}_{x y}=\hat{\varphi}_{x z}\right| \hat{\varphi}_{y z}^{-1} .
$$

The mapping $\tau_{x y z}^{-1}$ coincides with $\tau_{x z y}$, by (2), and $\hat{\varphi}_{y z}^{-1}$ coincides with $\hat{\varphi}_{z y}$, because, as has already been pointed out, semi-frame condition (ii) is valid in $\mathcal{F}$. The previous equation may therefore be rewritten in the form

$$
\hat{\varphi}_{x z}\left|\hat{\varphi}_{z y}=\tau_{x z y}\right| \hat{\varphi}_{x y},
$$

which is a permuted version of (4) in which the second and third indices $y$ and $z$ have been transposed. Compose both sides of (4) on the right with $\hat{\varphi}_{x z}^{-1}$ and on the left with $\hat{\varphi}_{x y}^{-1}$ to obtain

Observe that

$$
\hat{\varphi}_{y z}\left|\hat{\varphi}_{x z}^{-1}=\hat{\varphi}_{x y}^{-1}\right| \tau_{x y z} .
$$

$$
\hat{\varphi}_{x y}^{-1}\left|\tau_{x y z}=\hat{\varphi}_{x y}^{-1}\right| \tau_{x y z}\left|\hat{\varphi}_{x y}\right| \hat{\varphi}_{x y}^{-1}=\hat{\varphi}_{x y}^{-1}\left|\hat{\varphi}_{x y}\right| \tau_{y z x}\left|\hat{\varphi}_{x y}^{-1}=\tau_{y z x}\right| \hat{\varphi}_{x y}^{-1},
$$

by the properties of isomorphism composition and (3). It follows from these computations and from the validity of semi-frame condition (ii) in $\mathcal{F}$ that

$$
\hat{\varphi}_{y z}\left|\hat{\varphi}_{z x}=\hat{\varphi}_{y z}\right| \hat{\varphi}_{x z}^{-1}=\hat{\varphi}_{x y}^{-1}\left|\tau_{x y z}=\tau_{y z x}\right| \hat{\varphi}_{x y}^{-1}=\tau_{y z x} \mid \hat{\varphi}_{y x},
$$

which is a permuted version of (4) in which the indices have been shifted one to the left modulo 3 , so that $x, y$, and $z$ have been replaced by $y, z$, and $x$ respectively. This argument shows that the two permuted versions of (44), the first obtained by transposing the last two indices $y$ and $z$ of the triple $(x, y, z)$ to arrive at (5), and the second by shifting each of the indices $x, y$, and $z$ of the triple to the left by one modulo 3 to arrive at (6), are valid in $\mathcal{F}$. All permutations of the triple $(x, y, z)$ may be obtained by composing these two permutations. For example, transpose the last two indices of (4), permuting $(x, y, z)$ to $(x, z, y)$, to obtain (5), and then use (6) to shift the indices of (5) to the left by one modulo 3 , permuting $(x, z, y)$ to $(z, y, x)$, to arrive at

$$
\hat{\varphi}_{z y}\left|\hat{\varphi}_{y x}=\tau_{z y x}\right| \hat{\varphi}_{z y} .
$$

It follows that semi-frame condition (iv) is valid in $\mathcal{F}$. 
The next step in the proof is the verification of the coset conditions for the identity law, the second involution law, and the cycle law under the assumption of conditions (i)-(viii) of the theorem. Certainly, $\mathcal{F}$ will satisfy the coset condition for the identity law, since this is just the equality of the last two cosets in condition (v) of the theorem. In order to verify the coset conditions for the second involution law and the cycle law, which coincide with conditions (ii) and (iv) in Lemma 4.4 it suffices to show that conditions (i) and (v) of that lemma, namely

$$
C_{x y z}^{-1}=C_{x z y}
$$

and

$$
\varphi_{x y}\left[C_{x y z}\right]=C_{y z x},
$$

hold for all triples $(x, y, z)$ in $\mathcal{E}_{3}$. If two of the indices, say $x$ and $y$, are equal, then

$$
\begin{aligned}
& C_{x y z}=C_{x x z}=H_{x z}=\left\{e_{x}\right\} \circ H_{x z}=H_{x y} \circ H_{x z}, \\
& C_{x z y}=C_{x z x}=H_{x z}=H_{x z} \circ\left\{e_{x}\right\}=H_{x z} \circ H_{x y}, \\
& C_{z x y}=C_{z x x}=H_{z x}=H_{z x} \circ H_{z x}=H_{z x} \circ H_{z y},
\end{aligned}
$$

by the assumption on $x$ and $y$, condition ( $\mathrm{v}$ ) (with $z$ in place of $y$ ), condition (i), which implies that $H_{x y}=\left\{e_{x}\right\}$, and, for the second to the last equality in the last line, the assumption that $H_{z x}$ is a subgroup of $G_{z}$ and therefore closed under composition. It is clear from this argument that (12) holds for all permutations of the indices $x, y$, and $z$. Apply Lemma 4.5 to arrive at (10). The cases $y=z$ and $x=z$ are handled in a similar fashion.

As regards the verification of (11), if two of the indices, say $x$ and $y$ are equal, then (12) holds for all permutations of the variables $x, y$, and $z$, and therefore Lemma 4.5 yields (11). A similar argument applies if $y=z$ or $x=z$.

Assume now that all three indices $x, y$, and $z$ are distinct. If $x<y<z$, then (11) holds, by condition (vii) of the theorem. To derive the permuted version of (11) in which the indices $x$ and $y$ are transposed, use condition (vi), the isomorphism properties of $\varphi_{x y}$, condition (vii), and condition (vi) (with $y, z$, and $x$ in place of $x, y$, and $z$ respectively) to obtain

$$
\varphi_{x y}\left[C_{x z y}\right]=\varphi_{x y}\left[C_{x y z}^{-1}\right]=\varphi_{x y}\left[C_{x y z}\right]^{-1}=C_{y z x}^{-1}=C_{y x z} .
$$

Apply $\varphi_{y x}$ to the first and last terms in this string of equalities, and use the fact that $\varphi_{y x}$ is the inverse of $\varphi_{x y}$, by semi-frame condition (ii), to arrive at

$$
\varphi_{y x}\left[C_{y x z}\right]=C_{x z y} .
$$

To derive the permuted version of (11) in which $x, y$, and $z$ are shifted one to the right modulo 3 to obtain the equation for $z, x$, and $y$ respectively,

$$
\varphi_{z x}\left[C_{z x y}\right]=C_{x y z},
$$

apply $\varphi_{z x}$ to both sides of condition (viii), and use semi-frame condition (ii) (with $z$ in place of $y$ ).

The permutation of the triple $(x, y, z)$ implicit in (13) that is obtained by transposing the first two indices to obtain $(y, x, z)$, and the permutation of the triple implicit in (11) that is obtained by shifting each index to the right by one modulo 3 to obtain $(z, x, y)$, together generate all permutations of $(x, y, z)$, and hence all permutations of (11). For example, use (13) to shift all the indices of (11) to the 
right by one modulo 3 , permuting $(x, y, z)$ to $(z, x, y)$ and arriving at (14), and then repeat this process on (14), permuting $(z, x, y)$ to $(y, z, x)$, to arrive at

$$
\varphi_{y z}\left[C_{y z x}\right]=C_{z x y} .
$$

From these observations, it is clear that (11) holds for all permuted versions of a given triple of distinct elements in $\mathcal{E}_{3}$. Combine this with the arguments following (11) to see that (11) holds for all triples in $\mathcal{E}_{3}$. Use (10), (11), and Lemma 4.4 to conclude the coset conditions for the second involution law and the cycle law hold in $\mathcal{F}$. This completes the derivation of the coset conditions for the identity law, the second involution law, and the cycle law from conditions (i)-(viii) above.

To establish the reverse implication, assume $\mathcal{F}$ is a semi-frame satisfying the coset conditions for the identity law, the second involution law, and the cycle law. Certainly, $\mathcal{F}$ satisfies conditions (i)-(iv) of the theorem, because these conditions are special cases of the semi-frame conditions. To see that $\mathcal{F}$ satisfies condition (v), use the coset condition for the identity law for the pair $(y, x)$, which says that $C_{y x x}=H_{y x}$, use the definition of $\varphi_{y x}$, and use semi-frame condition (ii) in the form of Convention 2.6 (with $x$ and $y$ interchanged), to obtain

$$
\varphi_{y x}\left[C_{y x x}\right]=\varphi_{y x}\left[H_{y x}\right]=K_{y x}=H_{x y} .
$$

The coset conditions for the second involution law and the cycle law are conditions (ii) and (iv) of Lemma 4.4, so they imply all of the other conditions of the lemma. In particular, they imply (v) (with $y, x$, and $x$ in place of $x, y$, and $z$ respectively), so

$$
\varphi_{y x}\left[C_{y x x}\right]=C_{x x y} .
$$

Combine (15) and (16) to arrive at

$$
C_{x x y}=H_{x y} .
$$

Invoke Lemma 4.4 again, this time using (i) (with $x$ and $y$ in place of $y$ and $z$ respectively), to obtain

$$
C_{x x y}^{-1}=C_{x y x} .
$$

Combine this equation with (17), and use the fact that $H_{x y}$ is a subgroup of $G_{x}$ and therefore closed under inverse, to arrive at

$$
C_{x y x}=C_{x x y}^{-1}=H_{x y}^{-1}=H_{x y} .
$$

Together, the coset condition for the identity law, (17), and (18) imply condition (v) of the theorem. To derive conditions (vi), (vii), and (viii) of the theorem, use Lemma 4.4 again, and in fact parts (i), (v), and (iii) respectively. This completes the proof of the first assertion of the theorem.

To prove the second assertion of the theorem, suppose that $\mathcal{F}$ satisfies conditions (i)-(viii) of the theorem. It follows from the first part of the theorem that $\mathcal{F}$ must be a semi-frame that satisfies the first three coset conditions. The key step in the argument is showing that $\mathcal{F}$ satisfies the coset condition for the associative law for one quadruple of elements in $\mathcal{E}_{4}$ if and only if it satisfies the condition for every permutation of that quadruple.

Fix a quadruple $(x, y, z, w)$ in $\mathcal{E}_{4}$ of not necessarily distinct elements, and suppose that

$$
C_{x y z}{ }^{\circ} C_{x z w}=\varphi_{y x}\left[C_{y z w}{ }^{\circ} H_{y x}\right] \circ C_{x y w} .
$$


The immediate goal is to derive a permuted version of (19) in which the indices $z$ and $w$ have been transposed. Form the coset inverses of both sides of (19), and apply the second involution law for cosets, to obtain

$$
C_{x z w}^{-1}{ }^{\circ} C_{x y z}^{-1}=C_{x y w}^{-1}{ }^{\circ} \varphi_{y x}\left[C_{y z w}{ }^{\circ} H_{y x}\right]^{-1} .
$$

Conditions (ii) and (iv) in Lemma 4.4 hold for all triples of indices in $\mathcal{E}_{3}$, because $\mathcal{F}$ satisfies the coset conditions for the second involution law and the cycle law. Consequently, part (i) of the lemma holds for all such triples. Use it repeatedly on different triples to obtain

$$
C_{x y z}^{-1}=C_{x z y}, \quad C_{x y w}^{-1}=C_{x w y}, \quad C_{x z w}^{-1}=C_{x w z}, \quad C_{y z w}^{-1}=C_{y w z} .
$$

Expand the second term on the right side of (20) as follows:

$$
\begin{aligned}
\varphi_{y x}\left[C_{y z w}{ }^{\circ} H_{y x}\right]^{-1} & =\varphi_{y x}\left[\left(C_{y z w}{ }^{\circ} H_{y x}\right)^{-1}\right]=\varphi_{y x}\left[H_{y x}^{-1} \circ C_{y z w}^{-1}\right] \\
& =\varphi_{y x}\left[H_{y x}{ }^{\circ} C_{y z w}^{-1}\right]=\varphi_{y x}\left[C_{y z w}^{-1}{ }^{\circ} H_{y x}\right]=\varphi_{y x}\left[C_{y w z} \circ H_{y x}\right],
\end{aligned}
$$

by the isomorphism properties of $\varphi_{y x}$, the second involution law for cosets, the assumption that $H_{y z}$ is a normal subgroup of $G_{x}$, and hence is closed under inverses and commutes with all elements in $G_{x}$, and the final equation in (21). Combine (22) with (20) and the first three equations in (21) to arrive at

$$
\begin{aligned}
C_{x w z}{ }^{\circ} C_{x z y}=C_{x z w}^{-1}{ }^{\circ} C_{x y z}^{-1}=C_{x y w}^{-1}{ }^{\circ} \varphi_{y x}\left[C_{y z w}{ }^{\circ} H_{y x}\right]^{-1} & \\
& =C_{x w y}{ }^{\circ} \varphi_{y x}\left[C_{y w z} \circ H_{y x}\right] .
\end{aligned}
$$

Multiply the first and last expressions in (23) on the left by $C_{x w y}^{-1}$ and on the right by $C_{x z y}^{-1}$, and use the inverse law for cosets, to obtain

$$
C_{x w y}^{-1}{ }^{\circ} C_{x w z}=\varphi_{y x}\left[C_{y w z} \circ H_{y x}\right] \circ C_{x z y}^{-1} .
$$

In more detail, the inverse law for cosets, the assumption that $C_{x z y}$ is a coset of $H_{x z}{ }^{\circ} H_{x y}$, and the assumption that the subgroup $H_{x y}$ is normal yield

$$
\begin{aligned}
C_{x w y}^{-1}{ }^{\circ} C_{x w z}{ }^{\circ} C_{x z y}{ }^{\circ} C_{x z y}^{-1}=C_{x w y}^{-1}{ }^{\circ} C_{x w z}{ }^{\circ} H_{x z} \circ H_{x y} & \\
& =C_{x y w}^{-1}{ }^{\circ} H_{x y}{ }^{\circ} C_{x w z}{ }^{\circ} H_{x z}=C_{x y w}^{-1}{ }^{\circ} C_{x w z} .
\end{aligned}
$$

The final equality is justified because $C_{x w z}$ is a coset of the normal subgroup $H_{x w}{ }^{\circ} H_{x z}$, and therefore absorbs the factor $H_{x z}$ in the sense that

$$
C_{x w z} \circ H_{x z}=C_{x w z} \circ\left(H_{x w} \circ H_{x z}\right) \circ H_{x z}=C_{x w z} \circ H_{x w} \circ H_{x z}=C_{x w z},
$$

by the identity law for groups of cosets, the assumption that $C_{x w z}$ is a coset of $H_{x w}{ }^{\circ} H_{x z}$, and the assumption that $H_{x z}$ is a subgroup of $G_{x}$ and therefore closed under composition. Similarly, the coset $C_{x w y}^{-1}$ of $H_{x w}{ }^{\circ} H_{x y}$ absorbs the factor $H_{x y}$. An analogous argument shows that the product of $C_{x z y}$ with its inverse is absorbed by the term $\varphi_{y x}\left[C_{y w z}{ }^{\circ} H_{y x}\right]$ on the right side of equation (24). This completes the justification of the computation in (24). Combine the first and second equations in (21) with (24) to conclude that

$$
C_{x y w}{ }^{\circ} C_{x w z}=\varphi_{y x}\left[C_{y w z} \circ H_{y x}\right] \circ C_{x y z} .
$$

This is just the desired permuted version of (19) in which the indices $z$ and $w$ have been transposed. 
The next goal is to derive a permuted version of (19) in which the indices $y$ and $w$ have been transposed. Begin with an application of Lemma 3.6(iii) (with $w$ and $y$ in place of $y$ and $z$ respectively, and with $C_{y w z}{ }^{\circ} H_{y x}$ in place of $Q$ ) to obtain

$$
C_{x w y}{ }^{\circ} \varphi_{x y}^{-1}\left[C_{y w z}{ }^{\circ} H_{y z}\right]=\varphi_{x w}^{-1}\left[\varphi_{w y}^{-1}\left[C_{y w z}{ }^{\circ} H_{y x}\right]\right] \circ C_{x w y} .
$$

Notice in this connection that $C_{y w z}$ is a coset of $H_{y w}{ }^{\circ} H_{y z}$, so the product $C_{y w z}{ }^{\circ} H_{y x}$ is a coset of $H_{y w}{ }^{\circ} H_{y z} \circ H_{y x}$, and therefore a union of cosets of $H_{y w}{ }^{\circ} H_{y x}$. This latter group coincides with $K_{x y} \circ K_{w y}$, by semi-frame condition (ii) and Convention 2.6. so the hypotheses of Lemma 3.6(iii) are indeed satisfied. Use semi-frame condition (ii) to rewrite (26) as

$$
C_{x w y} \circ \varphi_{y x}\left[C_{y w z} \circ H_{y z}\right]=\varphi_{w x}\left[\varphi_{y w}\left[C_{y w z} \circ H_{y x}\right]\right] \circ C_{x w y} .
$$

The argument of $\varphi_{w x}$ on the right side of (27) may be rewritten as

$$
\begin{aligned}
\varphi_{y w}\left[C_{y w z} \circ H_{y x}\right] & =\varphi_{y w}\left[C_{y w z} \circ H_{y w} \circ H_{y x}\right] \\
& =\varphi_{y w}\left[C_{y w z}\right] \circ \varphi_{y w}\left[H_{y w} \circ H_{y x}\right] .
\end{aligned}
$$

The first equality uses the fact that $C_{y w z}$ is a coset of $H_{y w}{ }^{\circ} H_{y z}$ and therefore absorbs $H_{y w}$, and the second uses the isomorphism properties of $\varphi_{y w}$. The function $\varphi_{y w}$ maps the group $K_{x y}{ }^{\circ} H_{y w}$ to the group $K_{x w}{ }^{\circ} K_{y w}$, by the second equation in condition (iii) of the theorem (with $w$ in place of $z$ ), which has been shown to hold for all triples in $\mathcal{E}_{3}$. The first of these groups coincides with $H_{y x}{ }^{\circ} H_{y w}$, and the second with $H_{w x} \circ H_{w y}$, by semi-frame condition (ii) and Convention 2.6. so (using also the assumption that the subgroups involved are normal)

$$
\varphi_{y w}\left[H_{y w} \circ H_{y x}\right]=H_{w y} \circ H_{w x} .
$$

Also, parts (ii) and (iv) of Lemma 4.4 hold for all triples in $\mathcal{E}_{3}$, because $\mathcal{F}$ satisfies the coset conditions for the second involution law and the cycle law. Apply part (v) of the lemma (with $y$ and $w$ in place of $x$ and $y$ respectively) to obtain

$$
\varphi_{y w}\left[C_{y w z}\right]=C_{w z y} .
$$

Combine (28)-(30), and use the fact that the coset $C_{w z y}$ of $H_{w z}{ }^{\circ} H_{w y}$ absorbs the subgroup $H_{w y}$, to arrive at

$$
\begin{aligned}
\varphi_{y w}\left[C_{y w z} \circ H_{y x}\right]=\varphi_{y w}\left[C_{y w z}\right] \circ \varphi_{y w}\left[H_{y w} \circ H_{y x}\right] \\
\quad=C_{w z y} \circ H_{w y} \circ H_{w x}=C_{w z y} \circ H_{w x} .
\end{aligned}
$$

Replace the occurrence in (27) of the left side of (31) with the right side of (31) to get

$$
C_{x w y}{ }^{\circ} \varphi_{y x}\left[C_{y w z} \circ H_{y z}\right]=\varphi_{w x}\left[C_{w z y}{ }^{\circ} H_{w x}\right] \circ C_{x w y} .
$$

Combine this with (23) to conclude that

$$
C_{x w z}{ }^{\circ} C_{x z y}=\varphi_{w x}\left[C_{w z y} \circ H_{w x}\right] \circ C_{x w y},
$$

which is the permuted version of (19) in which the indices $y$ and $w$ have been transposed.

Finally, we derive a permuted version of (19) in which the indices $x$ and $y$ have been transposed. Apply $\varphi_{x y}$ to both sides of (19) to obtain

$$
\varphi_{x y}\left[C_{x y z} \circ C_{x z w}\right]=\varphi_{x y}\left[\varphi_{y x}\left[C_{y z w}{ }^{\circ} H_{y x}\right] \circ C_{x y w}\right] .
$$


The left side of (33) may be rewritten as

$$
\begin{aligned}
\varphi_{x y}\left[C_{x y z}{ }^{\circ} C_{x z w}\right]= & \varphi_{x y}\left[C_{x y z}{ }^{\circ} H_{x y}{ }^{\circ} C_{x z w}\right]=\varphi_{x y}\left[C_{x y z}{ }^{\circ} C_{x z w}{ }^{\circ} H_{x y}\right] \\
& =\varphi_{x y}\left[C_{x y z}\right] \circ \varphi_{x y}\left[C_{x z w}{ }^{\circ} H_{x y}\right]=C_{y z x}{ }^{\circ} \varphi_{x y}\left[C_{x z w} \circ H_{x y}\right] .
\end{aligned}
$$

The first equality uses the fact that the coset $C_{x y z}$ of $H_{x y}{ }^{\circ} H_{x z}$ absorbs the subgroup $H_{x y}$, the second uses the assumption that $H_{x y}$ is normal, the third uses the isomorphism properties of $\varphi_{x y}$ (which is why it is necessary to insert a copy of $H_{x y}$ to compose with $C_{x z w}$ ), and the fourth uses Lemma 4.4(v). The right side of (33) may be rewritten as

$$
\begin{aligned}
\varphi_{x y}\left[\varphi_{y x}\left[C_{y z w} \circ H_{y x}\right] \circ C_{x y w}\right] & =\varphi_{x y}\left[\varphi_{y x}\left[C_{y z w} \circ H_{y x}\right]\right] \circ \varphi_{x y}\left[C_{x y w}\right] \\
& =C_{y z w}{ }^{\circ} H_{y x} \circ \varphi_{x y}\left[C_{x y w}\right] \\
& =C_{y z w}{ }^{\circ} H_{y x} \circ C_{y w x} \\
& =C_{y z w}{ }^{\circ} C_{y w x}
\end{aligned}
$$

by isomorphism properties of $\varphi_{x y}$, semi-frame condition (ii), Lemma 4.4(v) (with $w$ in place of $z$ ), and the fact that the $\operatorname{coset} C_{y w x}$ absorbs the group $H_{y x}$. Combine (33) - (35) to arrive at

$$
C_{y z w}{ }^{\circ} C_{y w x}=C_{y z x}{ }^{\circ} \varphi_{x y}\left[C_{x z w}{ }^{\circ} H_{x y}\right] .
$$

Multiply both sides of the preceding equation by $C_{y z x}^{-1}$ on the left and by $C_{y w x}^{-1}$ on the right, and use the inverse law for groups of cosets, to obtain

$$
C_{y z x}^{-1}{ }^{\circ} C_{y z w}=\varphi_{x y}\left[C_{x z w}{ }^{\circ} H_{x y}\right]{ }^{\circ} C_{y w x}^{-1} .
$$

From Lemma 4.4(i), it follows that

$$
C_{y z x}^{-1}=C_{y x z} \quad \text { and } \quad C_{y w x}^{-1}=C_{y x w} .
$$

Combine (36) and (37) to conclude that

$$
C_{y x z}{ }^{\circ} C_{y z w}=\varphi_{x y}\left[C_{x z w}{ }^{\circ} H_{x y}\right] \circ C_{y x w},
$$

which is the desired permuted version of (19) obtained by transposing the indices $x$ and $y$.

It has been shown that the three permuted versions of (19) obtained by transposing the indices $z$ and $w$, the indices $y$ and $w$, and the indices $x$ and $y$, are all derivable from (19). These three transpositions generate all permutations of the quadruple $(x, y, z, w)$, so it follows that every version of (19) in which the indices $x, y, z$, and $w$ have been permuted is derivable from (19).

The next step is to derive all instances of the coset condition for the associative law on the basis of condition (ix) of the theorem and the assumption that $\mathcal{F}$ is a semi-frame satisfying conditions (i)-(viii) of the theorem, or equivalently, satisfying the first three coset conditions. Suppose that the first two indices of an arbitrary quadruple in $\mathcal{E}_{4}$, say $(x, y, z, w)$, are equal, with the goal of deriving (19). This derivation does not require the use of condition (ix) at all. Observe that

$$
C_{x y z}=C_{x x z}=H_{x z} \quad \text { and } \quad C_{x y w}=C_{x x w}=H_{x w},
$$

by the assumption on $x$ and $y$, and condition (v) of the theorem. Also, $\varphi_{y x}$ and $H_{y x}$ coincide with $\varphi_{x x}$ and $\left\{e_{x}\right\}$ respectively, and $\varphi_{x x}$ is the identity function on $G_{x} /\left\{e_{x}\right\}$, by condition (i) of the theorem, so

$$
\varphi_{y x}\left[C_{y z w} \circ H_{y x}\right]=\varphi_{x x}\left[C_{x z w} \circ\left\{e_{x}\right\}\right]=C_{x z w} \circ\left\{e_{x}\right\}=C_{x z w} .
$$


Consequently,

$$
C_{x y z}{ }^{\circ} C_{x z w}=H_{x z}{ }^{\circ} C_{x z w}=C_{x z w},
$$

by the first part of (39) and the fact that the $\operatorname{coset} C_{x z w}$ absorbs the subgroup $H_{x z}$. Therefore,

$$
\varphi_{y x}\left[C_{y z w} \circ H_{y z}\right] \circ C_{x y w}=C_{x z w}{ }^{\circ} C_{x y w}=C_{x z w}{ }^{\circ} H_{x w}=C_{x z w},
$$

by (40), the second part of (39), and the fact that the coset $C_{x z w}$ absorbs the subgroup $H_{x w}$. Combine (41) and (42) to arrive at (19).

Consider next the case of an arbitrary quadruple $(x, y, z, w)$ in $\mathcal{E}_{4}$ in which at least two of the indices are equal. Form a permutation of this quadruple in which two of the equal indices are moved to the first and second positions of the quadruple. The resulting quadruple satisfies the hypotheses of the preceding paragraph, so the version of (19) that is associated with this quadruple is valid in $\mathcal{F}$, by the observations of the previous paragraph. It follows that (19) must hold for the given quadruple $(x, y, z, w)$, since every permuted version of a valid instance of the coset condition for the associative law is also valid.

Turn finally to the case when the indices in a quadruple $(x, y, z, w)$ in $\mathcal{E}_{4}$ are distinct. If $x<y<z<w$, then (19) holds by the assumed condition (ix). Consequently, every permuted version of (19) also holds, so (19) is valid in $\mathcal{F}$ in all cases in which the indices of the given quadruple are mutually distinct. Combine the observations of this and the preceding paragraph to conclude that if condition (ix) of the theorem is true in a semi-frame $\mathcal{F}$ satisfying conditions (i)-(viii), then the coset condition for the associative law holds in $\mathcal{F}$. The reverse implication is trivially true.

The following special case of the second part of Theorem 4.6 is quite useful in verifying the coset condition for the associative law in basic examples of semi-frames.

Corollary 4.7. Suppose $\mathcal{F}$ is a semi-frame satisfying the coset conditions for the identity law, the second involution law, and the cycle law. If

$$
H_{x y} \circ H_{x z} \circ H_{x w}=G_{x}
$$

for all quadruples $(x, y, z, w)$ in $\mathcal{E}_{4}$, then $\mathcal{F}$ satisfies the coset conditions for the associative law.

Proof. Consider a quadruple $(x, y, z, w)$ in $\mathcal{E}_{4}$, with the intention of showing that

$$
C_{x y z}{ }^{\circ} C_{x z w}=\varphi_{y x}\left[C_{y z w}{ }^{\circ} H_{y x}\right]{ }^{\circ} C_{x y w} .
$$

Since $C_{x y z}$ and $C_{x z w}$ are cosets of $H_{x y}{ }^{\circ} H_{x z}$ and $H_{x z} \circ H_{x w}$, the complex product

$$
C_{x y z}{ }^{\circ} C_{x z w}
$$

is a coset of the triple product

$$
H_{x y}{ }^{\circ} H_{x z} \circ H_{x w},
$$

which is $G_{x}$, by assumption. There is only one coset of the improper subgroup $G_{x}$, namely itself, so

$$
C_{x y z}{ }^{\circ} C_{x z w}=G_{x} .
$$

As regards the right side of (1), because $C_{y z w}$ is a coset of $H_{y z} \circ H_{y w}$, the product

$$
C_{y z w}{ }^{\circ} H_{y x}
$$


is a coset of the triple product

$$
H_{y z} \circ H_{y w}{ }^{\circ} H_{y x},
$$

which is $G_{y}$, by assumption. Therefore,

$$
C_{y z w}{ }^{\circ} H_{y x}=G_{y} .
$$

Apply the mapping $\varphi_{y x}$ to both sides of the previous equation to obtain

$$
\varphi_{y x}\left[C_{y z w}{ }^{\circ} H_{y x}\right]=\varphi_{y x}\left[G_{y}\right]=G_{x} .
$$

Multiply the first and last terms of this equation on the right by $C_{x y w}$ to arrive at

$$
\varphi_{y x}\left[C_{y z w} \circ H_{y x}\right] \circ C_{x y w}=G_{x} \circ C_{x y w}=G_{x} .
$$

Combine (2) and (3) to see that (1) holds in this case. Apply Theorem 4.6 to conclude that coset conditions for the associative law are valid in $\mathcal{F}$.

There are a number of other special cases in which the verification of the coset conditions for a given semi-frame simplify. For instance, in many of the examples of group triples, most of cosets $C_{x y z}$ in the coset shifting system are the identity coset in the sense that they are the identity element of the corresponding quotient group,

$$
C_{x y z}=H_{x y}{ }^{\circ} H_{x z} .
$$

The next corollary is perhaps the simplest example of such a special case. Call two cosets $C_{x y z}$ and $C_{u v w}$ associated if $(u, v, w)$ is a permutation of $(x, y, z)$.

Corollary 4.8. Let $\mathcal{F}$ be a semi-frame, and $(p, q, r)$ a triple in $\mathcal{E}_{3}$ with $p<q<r$. If every coset not associated with $C_{\text {pqr }}$ is the identity coset, then $\mathcal{F}$ satisfies the four coset conditions if and only if the following conditions hold.

(i) $C_{p q r}^{-1}=C_{p r q}$, and $C_{q r p}^{-1}=C_{q p r}$, and $C_{r p q}^{-1}=C_{r q p}$.

(ii) $\varphi_{p q}\left[C_{p q r}\right]=C_{q r p}$.

(iii) $\varphi_{p r}\left[C_{p q r}\right]=C_{r p q}$.

(iv) $C_{p q r} \subseteq \bigcap\left\{H_{p q} \circ H_{p r} \circ H_{p s}:(p, s) \in \mathcal{E}\right.$ and $\left.s \neq p, q, r\right\}$.

Proof. Assume the conditions of the corollary, with the goal of verifying the conditions of Theorem 4.6. The assumption that $\mathcal{F}$ is a semi-frame implies that conditions (i) - (iv) of Theorem 4.6 are satisfied. Also, condition (v) of the theorem holds. To see this, consider an arbitrary pair $(x, y)$ in $\mathcal{E}$. The cosets

$$
C_{x x y}, \quad C_{x y y}, \quad C_{x y x},
$$

are identity cosets, by assumption, so

$$
C_{x x y}=H_{x x} \circ H_{x y}=\left\{e_{x}\right\} \circ H_{x y}=H_{x y}=H_{x y} \circ\left\{e_{x}\right\}=H_{x y} \circ H_{x x}=C_{x y x}
$$

and

$$
C_{x y y}=H_{x y}{ }^{\circ} H_{x y}=H_{x y} .
$$

The second and fifth equalities use semi-frame condition (i).

To verify that condition (vi) of the theorem is equivalent to condition (i) of the corollary (under the basic assumption of the corollary), let $(x, y, z)$ be a triple in $\mathcal{E}_{3}$ of pairwise distinct elements. If $(x, y, z)$ is not associated with $(p, q, r)$, then

$$
C_{x y z}^{-1}=\left(H_{x y} \circ H_{x z}\right)^{-1}=H_{x z}^{-1} \circ H_{x y}^{-1}=H_{x z} \circ H_{x y}=C_{x z y} .
$$

The first and last equality use the basic assumption of the corollary, the second uses the second involution law for group complexes, and the third uses the fact that $H_{x y}$ 
and $H_{x z}$ are subgroups, and hence closed under the operation of forming inverses. If $(x, y, z)$ is an associate of $(p, q, r)$, then condition (vi) of the theorem holds by condition (i) of the corollary, and vice versa.

The next step is to check that conditions (vii) and (viii) of the theorem are respectively equivalent to conditions (ii) and (iii) of the corollary. Let $(x, y, z)$ be a triple in $\mathcal{E}_{3}$ with $x<y<z$. If this triple is not $(p, q, r)$, then it cannot be an associate of $(p, q, r)$, because of the ordering, and therefore

$$
\varphi_{x y}\left[C_{x y z}\right]=\varphi_{x y}\left[H_{x y} \circ H_{x z}\right]=K / H=H_{y x} \circ H_{y z}=H_{y z} \circ H_{y x}=C_{y z x} .
$$

The first and fifth equalities hold by the basic assumption of the corollary, the second by semi-frame condition (iii), the third by semi-frame condition (ii) (and semi-frame condition (i) in the case when $x=y$ ), and the fourth by the fact that the subgroups are normal and hence commute with one another. A completely analogous argument shows that

$$
\varphi_{x z}\left[C_{x y z}\right]=C_{z x y} .
$$

Thus, in this case, conditions (vii) and (viii) of the theorem hold. If the triple $(x, y, z)$ is $(p, q, r)$, then conditions (vii) and (viii) of the theorem are exactly conditions (ii) and (iii) of the corollary.

The associative law coset conditions will hold for all permutations of a quadruple $(x, y, z, w)$ just in case

$$
C_{x y z}{ }^{\circ} C_{x z w}=\varphi_{y x}\left[C_{y z w}{ }^{\circ} H_{y x}\right] \circ C_{x y w},
$$

by Associative Law Theorem 3.8. By assumption,

$$
C_{x y w}=H_{x y} \circ H_{x w}, \quad C_{x z w}=H_{x z} \circ H_{x w}, \quad C_{y z w}=H_{y z} \circ H_{y w}
$$

(under the hypothesis that $w$ is different from $p, q$, and $r$ ), so equation (11) can equivalently be rewritten as

$$
C_{x y z} \circ H_{x z} \circ H_{x w}=\varphi_{y x}\left[H_{y z} \circ H_{y w} \circ H_{y x}\right] \circ H_{x y} \circ H_{x w} .
$$

It is a consequence of semi-frame condition (iii) that

$$
\varphi_{y x}\left[H_{y z} \circ H_{y w} \circ H_{y x}\right]=H_{x y} \circ H_{x z} \circ H_{x w},
$$

so the right-hand side of (2) reduces to $H_{x y}{ }^{\circ} H_{x z} \circ H_{x w}$. On the other hand,

$$
C_{x y z}{ }^{\circ} H_{x z}=C_{x y z},
$$

since $C_{x y z}$ is a coset of $H_{x y} \circ H_{x z}$, so the left-hand side of (2) reduces to $C_{x y z} \circ H_{x w}$. Thus, (2) is equivalent to

$$
C_{x y z}{ }^{\circ} H_{x w}=H_{x y}{ }^{\circ} H_{x z} \circ H_{x w} .
$$

Finally, since $C_{x y z}$ is a coset of $H_{x y}{ }^{\circ} H_{x z}$, equation (3) will hold just in case $C_{x y z}$ is a subset of $H_{x y}{ }^{\circ} H_{x z} \circ H_{x w}$.

If $(x, y, z)$ is not an associate of $(p, q, r)$, then $C_{x y z}$ is the identity coset $H_{x y}{ }^{\circ} H_{x z}$, and so the desired inclusion is trivial. If $(x, y, z)$ is an associate of $(p, q, r)$, then of course $(p, q, r)$ is an associate of $(x, y, z)$, and for $(p, q, r)$, the desired inclusion holds by condition (iv) of the corollary. This means that condition (1) holds for $(p, q, r)$, and hence also for the original triple $(x, y, z)$, since the validity of (11) for one triple implies its validity for all associates of the triple.

The remaining parts of the proof are trivial and are left to the reader. 
The final observation we wish to make is that in a coset relation algebra $\mathfrak{C}[\mathcal{F}]$, the operation $\otimes$ reduces to relational composition in all those cases in which the indices $x, y$, and $z$ of the coset $C_{x y z}$ used to define the relative product $R_{x y, \alpha} \otimes R_{y z, \beta}$ are not mutually distinct.

Corollary 4.9. If $\mathcal{F}$ is a group triple satisfying conditions (i)-(viii) of Theorem 4.6. then

$$
R_{x y, \alpha} \otimes R_{y z, \beta}=R_{x y, \alpha} \mid R_{y z, \beta}
$$

for every triple $(x, y, z)$ in $\mathcal{E}_{3}$ in which at least two of the indices $x, y, z$ are equal.

Proof. According to Lemma 3.10.

$$
R_{x y, \alpha} \otimes R_{y z, \beta}=R_{x y, \alpha} \mid R_{y z, \beta}
$$

if and only if

$$
C_{x y z}=H_{x y} \circ H_{x z} .
$$

The verification that (11) follows from conditions (i)-(viii) of Theorem 4.6 is nearly identical to the argument establishing (12) in the proof of Theorem 4.6. The details are left to the reader.

\section{EXAMPLE}

In this section, and example of a coset relation algebra that is not representable is constructed. Start with a group pair

$$
\mathcal{F}=(G, \varphi)=\left(\left\langle G_{x}: x \in I\right\rangle,\left\langle\varphi_{x y}:(x, y) \in I \times I\right\rangle\right)
$$

in which the index set $I$ has five elements, say

$$
I=\{p, q, r, s, t\} .
$$

Each of the groups $G_{x}$ is assumed to be a copy of the Cartesian product $\mathbb{Z}_{2} \times \mathbb{Z}_{2} \times \mathbb{Z}_{2}$, where $\mathbb{Z}_{2}=\{0,1\}$ denotes the cyclic group of order two, and these copies are assumed to be mutually disjoint. To describe the subgroups $H_{x y}$ and $K_{x y}$ for

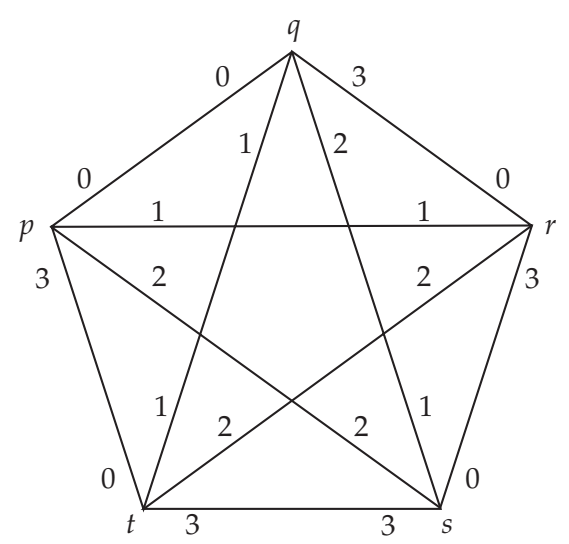

FiguRE 1. Normal subgroup diagram. 
distinct indices $x$ and $y$ in $I$, consider the following four subgroups of $\mathbb{Z}_{2} \times \mathbb{Z}_{2} \times \mathbb{Z}_{2}$ :

$$
\begin{array}{ll}
L_{0}=\mathbb{Z}_{2} \times\{0\} \times\{0\}, & L_{1}=\{0\} \times \mathbb{Z}_{2} \times\{0\}, \\
L_{2}=\{0\} \times\{0\} \times \mathbb{Z}_{2}, & L_{3}=\{(0,0,0),(1,1,1)\} .
\end{array}
$$

Take $H_{x y}$, respectively $K_{x y}$, to be the copy of one of these four subgroups in $G_{x}$, respectively $G_{y}$, according to the prescriptions given in Figure 1. For example, the subgroup $H_{p t}$ is the copy of $L_{3}$ in $G_{p}$ and the subgroup $K_{p t}$ is the copy of $L_{0}$ in $G_{t}$, because the edge between the vertices $p$ and $t$ in the diagram is labeled with 3 and 0. Similarly, the subgroup $H_{q s}$ is the copy of $L_{2}$ in $G_{q}$ and the subgroup $K_{q s}$ is the copy of $L_{1}$ in $G_{s}$, because the edge from $q$ to $s$ is labeled with 2 and 1.

The quotient isomorphisms $\varphi_{x y}$ when $x$ and $y$ are equal are of course taken to be the appropriate identity automorphisms of $G_{x} /\left\{e_{x}\right\}$ for every $x$ in $I$. For distinct $x$ and $y$, they are completely determined by the requirement that $\hat{\varphi}_{x y} \mid \hat{\varphi}_{y z}=\hat{\varphi}_{x z}$. For instance, according to the diagram in Figure 1, we must have

$$
\varphi_{p q}\left[L_{0} \circ L_{3}\right]=L_{0} \circ L_{1}, \quad \varphi_{p q}\left[L_{0} \circ L_{1}\right]=L_{0} \circ L_{3}, \quad \varphi_{p q}\left[L_{0} \circ L_{2}\right]=L_{0} \circ L_{2}
$$

(see (a), (b), and (c) respectively in Figure 2). (The composite subgroups on the

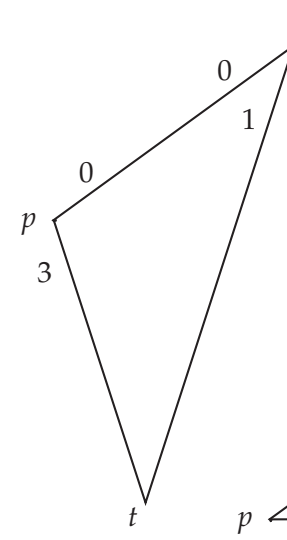

(a)

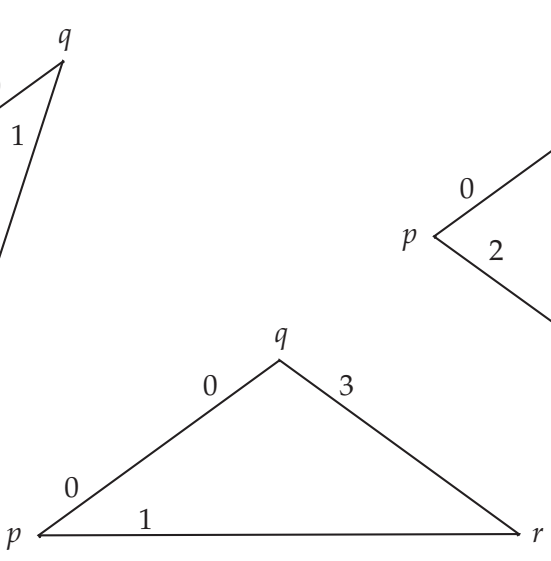

(b)

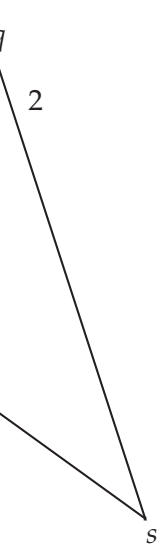

(c)

FiguRE 2. The triangles from the pentagon that determine $\varphi_{p q}$.

left, inside the brackets, should actually be interpreted as denoting their copies in $G_{p}$, and the composite subgroups on the right should be interpreted as denoting their copies in $G_{q}$.) These three requirements determine $\varphi_{p q}$ in the following way. According to the pentagon, the copy of the subgroup $L_{0}$ in $G_{p}$ is mapped by $\varphi_{p q}$ to the copy of the subgroup $L_{0}$ in $G_{q}$. The subgroup $L_{0}$ has four cosets in $\mathbb{Z}_{0} \times \mathbb{Z}_{0} \times \mathbb{Z}_{0}$, namely

$$
\begin{array}{ll}
C_{0}=(0,0,0) \circ L_{0}=\{(0,0,0),(1,0,0)\}, & C_{1}=(0,1,0) \circ L_{0}=\{(0,1,0),(1,1,0)\}, \\
C_{2}=(0,0,1) \circ L_{0}=\{(0,0,1),(1,0,1)\}, & C_{3}=(0,1,1) \circ L_{0}=\{(0,1,1),(1,1,1)\} .
\end{array}
$$


Observe that

$$
\begin{aligned}
L_{0} \circ L_{3} & =\{(0,0,0),(1,0,0)\} \circ\{(0,0,0),(1,1,1)\} \\
& =\{(0,0,0),(1,0,0),(0,1,1),(1,1,1)\}=C_{0} \cup C_{3}, \\
L_{0} \circ L_{1} & =\{(0,0,0),(1,0,0)\} \circ\{(0,0,0),(0,1,0)\} \\
& =\{(0,0,0),(1,0,0),(0,1,0),(1,1,0)\}=C_{0} \cup C_{1}, \\
L_{0} \circ L_{2} & =\{(0,0,0),(1,0,0)\} \circ\{(0,0,0),(0,0,1)\} \\
& =\{(0,0,0),(1,0,0),(0,0,1),(1,0,1)\}=C_{0} \cup C_{2} .
\end{aligned}
$$

Because $\varphi_{p q}$ maps the copies of $L_{0}{ }^{\circ} L_{3}$ and $C_{0}$ in $G_{p}$ respectively to the copies of $L_{0}{ }^{\circ} L_{1}$ and $C_{0}$ in $G_{q}$, it must map the copy of $C_{3}$ in $G_{p}$ to the copy of $C_{1}$ in $G_{q}$, by the preceding observations. Similarly, it must map the copies of $C_{1}$ and $C_{2}$ in $G_{p}$ respectively to the copies of $C_{3}$ and $C_{2}$ in $G_{q}$.

The resulting group pair $\mathcal{F}$ is easily seen to be a frame, so the group relation algebra $\mathfrak{G}[\mathcal{F}]$ exists. The next step is to modify the operation of relative multiplication in $\mathfrak{G}[\mathcal{F}]$ by introducing a coset system

$$
C=\left\langle C_{x y z}:(x, y, z) \in I \times I \times I\right\rangle .
$$

If a triple of indices $(x, y, z)$ is not a permutation of the triple $(p, q, r)$, take $C_{x y z}$ to be the identity coset,

$$
C_{x y z}=H_{x y} \circ H_{x z} \cdot
$$

Suppose now that $(x, y, z)$ is a permutation of $(p, q, r)$. As is clear from Figure 1, two different edges emanating from a given vertex $x$ are labeled with distinct numbers, so the subgroup $H_{x y}{ }^{\circ} H_{x z}$ is a composition of two distinct subgroups of $G_{x}$ of order 2 , and therefore has order 4 . It follows that the quotient group

$$
G_{x} /\left(H_{x y} \circ H_{x z}\right)
$$

has order 2 , so it has exactly two cosets, the identity coset and the non-identity coset. Take $C_{x y z}$ to be the non-identity coset,

$$
C_{x y z}=G_{x} \sim\left(H_{x y} \circ H_{x z}\right) .
$$

It is not difficult to check that the resulting group triple

$$
\overline{\mathcal{F}}=(G, \varphi, C)
$$

is a coset semi-frame that satisfies the coset conditions. For example, the quotient group in (1) is abelian, so the inner automorphism of (1) determined by the coset $C_{x y z}$ must be the identity automorphism. Use, in addition, the fact that $\mathcal{F}$ is a group frame to verify semi-frame condition (iv) for $\overline{\mathcal{F}}$,

$$
\hat{\varphi}_{x y}\left|\hat{\varphi}_{y z}=\hat{\varphi}_{x z}=\tau\right| \hat{\varphi}_{x z}
$$

The proof that $\overline{\mathcal{F}}$ satisfies the coset conditions is based on Corollary 4.8. It suffices to check that conditions (i)-(iv) of that corollary are satisfied. As regards condition (i), the quotient group in (1) has order 2 , so every coset is its own inverse. Consequently,

$$
C_{p q r}^{-1}=C_{p q r}=C_{p r q}=G_{p} \sim\left(H_{p q} \circ H_{p r}\right)
$$

and similarly

$$
C_{q r p}^{-1}=C_{q p r} \quad \text { and } \quad C_{r p q}^{-1}=C_{r q p}
$$


As regards conditions (ii) and (iii), the quotient isomorphisms $\hat{\varphi}_{p q}$ and $\hat{\varphi}_{p r}$ induced by $\varphi_{p q}$ and $\varphi_{p r}$ respectively map the identity coset to the identity coset, and consequently they map the non-identity coset to the non-identity coset. It follows that

$$
\begin{array}{r}
\varphi_{p q}\left[C_{p q r}\right]=\varphi_{p q}\left[G_{p} \sim\left(H_{p q} \circ H_{p r}\right)\right]=G_{q} \sim\left(K_{p q} \circ H_{q r}\right) \\
\quad=G_{q} \sim\left(H_{q p} \circ H_{q r}\right)=C_{q r p},
\end{array}
$$

and similarly, $\varphi_{p r}\left[C_{p q r}\right]=C_{r p q}$. Finally, to verify condition (iv) of the corollary, observe that each of the four edges emanating from vertex $p$ in Figure 1 is labeled with a different number. Consequently, the composite subgroups

$$
H_{p q} \circ H_{p r} \circ H_{p w}
$$

for $w=s, t$ have order 8 , that is to say, they coincide with $G_{p}$. The coset $C_{p q r}$ is trivially included in their intersection, since

$$
\left(H_{p q} \circ H_{p r} \circ H_{p s}\right) \cap\left(H_{p q} \circ H_{p r} \circ H_{p t}\right)=G_{p} .
$$

Apply Corollary 4.8 to arrive at the following conclusion.

Theorem 5.1. The group triple $\overline{\mathcal{F}}$ is a coset semi-frame that satisfies the coset conditions. Consequently, the corresponding algebra $\mathfrak{C}[\overline{\mathcal{F}}]$ is a full coset relation algebra and hence an example of a finite, measurable relation algebra.

It is instructive to look somewhat closer at the operation $\otimes$ of relative multiplication in the algebra $\mathfrak{C}[\overline{\mathcal{F}}]$ just constructed, and to compare it with the corresponding operation in $\mathfrak{G}[\mathcal{F}]$. On atoms, $\otimes$ is determined by

$$
R_{x y, a} \otimes R_{w z, \beta}=R_{x y, a} \mid R_{w z, \beta}
$$

whenever $y \neq w$, or $y=w$ and $\{x, y, z\} \neq\{p, q, r\}$, and

$$
R_{x y, a} \otimes R_{y z, \beta}=G_{x} \times G_{y} \sim\left(R_{x y, a} \mid R_{y z, \beta}\right)
$$

whenever $\{x, y, z\}=\{p, q, r\}$. Thus, the operation of relative multiplication in $\mathfrak{C}[\overline{\mathcal{F}}]$ is obtained by changing only slightly the operation of relational composition in $\mathfrak{G}[\mathcal{F}]$ as it affects atomic relations, namely, for those pairs of atomic relations $R_{x y, a}$ and $R_{y z, \beta}$ that are indexed, in some order, by a permutation $(x, y, z)$ of the triple $(p, q, r)$, the relative product has been shifted to the complement of what it is in $\mathfrak{G}[\mathcal{F}]$.

It turns out that the full coset relation algebra of the theorem is not representable as a set relation algebra, and in particular, it is not isomorphic to a full group relation algebra.

Theorem 5.2. The finite measurable relation algebra $\mathfrak{C}[\overline{\mathcal{F}}]$ is not representable.

Proof. Write $\mathfrak{A}=\mathfrak{C}[\overline{\mathcal{F}}]$. The argument that $\mathfrak{A}$ is not representable proceeds by contradiction. Assume that it is representable, say $\vartheta$ is a representation of $\mathfrak{A}$ over a base set $V$. Because $\mathfrak{A}$ is simple in the algebraic sense of the word (see the remarks preceding Theorem 6.1 below), it may be assumed that the unit of the representation is the Cartesian square $V \times V$ (see, for example, Theorem 16.18 in [3). We identify $R_{x x, 0}$ with $x$ in the proof, so that the set $I$ becomes the set of measurable atoms of $\mathfrak{A}$. This permits some simplification in the notation. 
The first step is to use the representation $\vartheta$ for constructing a scaffold in $\mathfrak{A}$, that is to say, a system of atoms $\left\langle a_{x y}: x, y \in I\right\rangle$ satisfying the following three conditions for all measurable atoms $x, y$, and $z$ in $I$.

$$
\begin{aligned}
& a_{x x}=x . \\
& a_{y x}=a_{x y}^{\smile} . \\
& a_{x z} \leq a_{x y} \otimes a_{y z} .
\end{aligned}
$$

Each element $x$ in $I$ is a subidentity atom, so its image $\vartheta(x)$ must be $i d_{V_{x}}$ for some non-empty subset $V_{x}$ of $V$, these sets are mutually disjoint for distinct $x$, and because $\mathfrak{A}$ is finite,

$$
\bigcup\left\{i d_{V_{x}}: x \in I\right\}=\bigcup\{\vartheta(x): x \in I\}=\vartheta\left(\sum I\right)=\vartheta\left(1^{\prime}\right)=i d_{V}
$$

For each $x$ in $I$, choose an element $v_{x}$ in $V_{x}$, and for each pair of elements $x$, let $a_{x y}$ be the unique atom in $\mathfrak{A}$ such that

$$
\left(v_{x}, v_{y}\right) \in \vartheta\left(a_{x y}\right) .
$$

Since $\vartheta(x)$ is the unique atom containing $\left(v_{x}, v_{x}\right)$, property (1) follows. Since $\vartheta\left(a_{x y}\right)$ is an atom (the converse of an atom is an atom) that contains $\left(v_{y}, v_{x}\right)$, by the representation properties of $\vartheta$, property (2) follows. Since $\left(v_{x}, v_{y}\right)$ is in $\vartheta\left(a_{x y}\right)$ and $\left(v_{y}, v_{z}\right)$ is in $\vartheta\left(a_{y z}\right)$, it follows from the definition of relational composition that $\left(v_{x}, v_{z}\right)$ is in $\vartheta\left(a_{x y}\right) \mid \vartheta\left(a_{y z}\right)$. The representation properties of $\vartheta$ imply that

$$
\vartheta\left(a_{x y}\right) \mid \vartheta\left(a_{y z}\right)=\vartheta\left(a_{x y} \otimes a_{y z}\right) .
$$

Thus, $\vartheta\left(a_{x z}\right)$ and $\vartheta\left(a_{x y} \otimes a_{y z}\right)$ have a non-empty intersection - they both contain the pair $\left(v_{x}, v_{z}\right)$ - so the former, which is an atom, must be below the latter. Use the representation properties of $\vartheta$ one more time to conclude that (3) holds. This completes the proof of the three scaffold conditions.

Here are some further properties of the elements $a_{x y}$ that we shall need. Notice that each such atom is actually one of the atomic binary relations of $\mathfrak{A}$ on the base set $U=\bigcup\left\{G_{x}: x \in I\right\}$, so it makes sense to speak of the pairs in $a_{x y}$. The converse of each atom is the set-theoretic relational inverse, in symbols,

$$
a_{y x}=a_{x y}^{-1} \text {. }
$$

Second, the relative product of two elements is the set-theoretic relation composition of the elements as long as the set of indices $\{x, y, z\}$ does not coincide with the set $\{p, q, r\}$,

$$
a_{x y} \otimes a_{y z}=a_{x y} \mid a_{y z} .
$$

Third, the relative product is disjoint from the relational composition when the two sets of indices $\{x, y, z\}$ and $\{p, q, r\}$ are equal,

$$
a_{x y} \otimes a_{y z}=G_{x} \times G_{y} \sim a_{x y} \mid a_{y z} .
$$

Fourth, the intersection of certain relative products that share a common "edge" is an atom when that common edge is $p q$ or $q r$ or $p r$. Specifically,

$$
\left(a_{p s} \otimes a_{s q}\right) \cap\left(a_{p t} \otimes a_{t q}\right)=a_{p q},
$$

and similarly if $p q$ is replaced by either $q r$ or $p r$.

Choose elements $u_{s}$ and $u_{t}$ in $U$ so that

$$
\left(u_{s}, u_{t}\right) \in a_{s t} .
$$


Such a choice is possible because $a_{s t}$ is a non-empty binary relation. Since for each $x=p, q, r$

$$
a_{s t} \leq a_{s x} \mid a_{x t},
$$

by (3) and (5), the pair in (8) must also belong to the the right side of (9), so that there must be an element $u_{x}$ in $U$ for which

$$
\left(u_{s}, u_{x}\right) \in a_{s x} \quad \text { and } \quad\left(u_{x}, u_{t}\right) \in a_{x t},
$$

by (8). In particular, take $x=p, q$, and use (44), to obtain

$$
\left(u_{p}, u_{s}\right) \in a_{p s} \quad \text { and } \quad\left(u_{s}, u_{q}\right) \in a_{s q},
$$

so that

$$
\left(u_{p}, u_{q}\right) \in a_{p s} \mid a_{s q}=a_{p s} \otimes a_{s q},
$$

and also to obtain

$$
\left(u_{p}, u_{t}\right) \in a_{p t} \quad \text { and } \quad\left(u_{t}, u_{q}\right) \in a_{t q}
$$

so that

$$
\left(u_{p}, u_{q}\right) \in a_{p t} \mid a_{t q}=a_{p t} \otimes a_{t q} .
$$

Apply (7) to arrive at

$$
\left(u_{p}, u_{q}\right) \in a_{p q} .
$$

Similar arguments applied to $p$ and $r$ and to $r$ and $q$ lead to

$$
\left(u_{p}, u_{r}\right) \in a_{p r} \quad \text { and } \quad\left(u_{r}, u_{q}\right) \in a_{r q} .
$$

In view of the definition of relational composition, (11) implies that

$$
\left(u_{p}, u_{q}\right) \in a_{p r} \mid a_{r q} .
$$

Together, (10) and (12) show that the intersection

$$
a_{p q} \cap\left(a_{p r} \mid a_{r q}\right)
$$

is not empty, since both factors contain the pair $\left(u_{p}, u_{q}\right)$. The left-hand factor is an atom, so

$$
a_{p q} \subseteq a_{p r} \mid a_{r q} .
$$

On the other hand,

$$
a_{p q} \subseteq a_{p r} \otimes a_{r q}=G_{p} \times G_{q} \sim a_{p r} \mid a_{r q},
$$

by (3) and (6). This is a direct contradiction to (13), so the assumption that $\mathfrak{A}$ is representable cannot be tenable.

The group $\mathbb{Z}_{2}$ can be replaced everywhere in the preceding construction by an arbitrary non-trivial abelian group. The mappings $\varphi_{x y}$ are no longer uniquely determined, and the definition of relative multiplication is slightly more involved. In each case we get an atomic, measurable relation algebra that is not representable. These are new examples of non-representable relation algebras, with a completely different underlying motivation than the examples that have appeared so far in the literature. 


\section{A Decomposition Theorem}

The isomorphism index set $\mathcal{E}$ of a coset semi-frame $\mathcal{F}=(G, \varphi, C)$ satisfying the coset conditions is an equivalence relation on the group index set $I$, and the unit

$$
E=\bigcup\left\{G_{x} \times G_{y}:(x, y) \in \mathcal{E}\right\}
$$

of the corresponding full coset relation algebra $\mathfrak{C}[\mathcal{F}]$ is an equivalence relation on the base set $U=\bigcup_{x \in I} G_{x}$. Call the semi-frame $\mathcal{F}$ simple if the group index set $I$ is not empty, and if $\mathcal{E}$ is the universal relation on the index set $I$. It turns out that $\mathcal{F}$ is simple in this sense of the word if and only if the algebra $\mathfrak{C}[\mathcal{F}]$ is simple in the algebraic sense of the word, namely, it has more than one element and every nonconstant homomorphism on the algebra is injective; or, equivalently, the algebra has exactly two ideals, the trivial ideal and the improper ideal.

Theorem 6.1. Let $\mathcal{F}$ be a semi-frame satisfying the coset conditions. The coset relation algebra $\mathfrak{C}[\mathcal{F}]$ is simple if and only if the semi-frame $\mathcal{F}$ is simple.

Proof. We begin with a preliminary observation: for all triples $(x, y, z)$ in $\mathcal{E}_{3}$,

$$
\bigcup\left\{R_{x y, \alpha} \otimes R_{y z, \beta}: \alpha<\kappa_{x y} \text { and } \beta<\kappa_{y z}\right\}=G_{x} \times G_{z} .
$$

For the proof, suppose that $(x, y, z)$ is in $\mathcal{E}_{3}$. The definition of $\otimes$ implies that

$$
R_{x y, \alpha} \otimes R_{y z, \beta}=\bigcup\left\{R_{x z, \gamma}: H_{x z, \gamma} \subseteq \varphi_{x y}^{-1}\left[K_{x y, \alpha}{ }^{\circ} H_{y z, \beta}\right]{ }^{\circ} C_{x y z}\right\} .
$$

Each relation $R_{x z, \gamma}$ is included in

$$
G_{x} \times G_{z}
$$

by Partition Lemma 2.2, so each product of the form (2) is included in (3), and therefore the left side of (11) is included in the right side.

To establish the reverse inclusion, notice that as the indices $\alpha$ and $\beta$ vary, the complex products $K_{x y, \alpha}{ }^{\circ} H_{y z, \beta}$ run through all cosets of the subgroup $K / H$. The function $\varphi_{x y}$ induces an isomorphism from the quotient group $G_{x} /\left(H_{x y} \circ H_{x z}\right)$ to the quotient group $G_{y} /(K / H)$, so the inverse images $\varphi_{x y}^{-1}\left[K_{x y, \alpha} \circ H_{y z, \beta}\right]$ must run through all of the cosets of $H_{x y}{ }^{\circ} H_{x z}$. It follows that, as $\alpha$ and $\beta$ vary, the complex products

$$
\varphi_{x y}^{-1}\left[K_{x y, \alpha}{ }^{\circ} H_{y z, \beta}\right] \circ C_{x y z}
$$

must also run through all cosets of $H_{x y}{ }^{\circ} H_{x z}$, because $C_{x y z}$ is a fixed element of the quotient group $G_{x} /\left(H_{x y}{ }^{\circ} H_{x z}\right)$. Thus, for each index $\gamma<\kappa_{x z}$, there are indices $\alpha<\kappa_{x y}$ and $\beta<\kappa_{y z}$ such that the coset $H_{x z, \gamma}$ of $H_{x z}$ is included in (44). The relation $R_{x z, \gamma}$ is therefore included in $R_{x y, \alpha} \otimes R_{y z, \beta}$, by (2). The union of all of the relations $R_{x z, \gamma}$ is (3), by Partition Lemma 2.2, so the right side of (10) must be included in the left side.

Turn now to the proof of the theorem, and assume first that the semi-frame $\mathcal{F}$ is simple. The isomorphism index set $\mathcal{E}$ is the universal relation on the group index set $I$, by assumption, so

$$
\begin{aligned}
U \times U & =\left(\bigcup_{x \in I} G_{x}\right) \times\left(\bigcup_{y \in I} G_{y}\right)=\bigcup\left\{G_{x} \times G_{y}: x, y \in U\right\} \\
& =\bigcup\left\{R_{x y, \alpha}: x, y \in U \text { and } \alpha<\kappa_{x y}\right\}=\bigcup\left\{G_{x} \times G_{y}:(x, y) \in \mathcal{E}\right\}=E,
\end{aligned}
$$

by the definition of $U$, the distributivity of Cartesian products over arbitrary unions, Partition Lemma 2.2. the assumption on $\mathcal{E}$, and the definition of $E$. The index set $I$ is assumed to be non-empty, and the groups are non-empty, so the unit $U \times U$ of 
$\mathfrak{C}[\mathcal{F}]$ is non-empty and therefore different from the zero element $\varnothing$. In particular, the relation algebra $\mathfrak{C}[\mathcal{F}]$ has more than one element.

In order to show that a non-degenerated, atomic relation algebra is simple, it suffices to show that the equation $1 ; r ; 1=1$ holds for every subidentity atom $r$ (see, for example, Givant [2], Theorem 9.2). A subidentity atom of $\mathfrak{C}[\mathcal{F}]$ has the form $R_{y y, 0}$ for some $y$ in $I$, so it must be shown that

$$
(U \times U) \otimes R_{y y, 0} \otimes(U \times U)=U \times U
$$

for every $y$ in $I$. Use (5) and the distributivity of $\otimes$ over arbitrary unions to rewrite the left side of (6) as the union of the relations

$$
R_{x u, \alpha} \otimes R_{y y, 0} \otimes R_{v z, \beta}
$$

over all $x, u, v, z$ in $I$, with $\alpha<\kappa_{x u}$ and $\beta<\kappa_{v z}$. If $u \neq y$ or $v \neq y$, then the relation in (7) reduces to the empty relation, by the definition of $\otimes$. The left side of (6) is therefore equal to the union of the relations

$$
R_{x y, \alpha} \otimes R_{y y, 0} \otimes R_{y z, \beta}
$$

over all $x$ and $z$ in $I$, with $\alpha<\kappa_{x y}$ and $\beta<\kappa_{y z}$. The coset condition for the identity law, which $\mathcal{F}$ is assumed to satisfy, and Identity Law Theorem 3.4. imply that

$$
R_{x y, \alpha} \otimes R_{y y, 0}=R_{x y, \alpha} .
$$

Consequently, (8) reduces to

$$
R_{x y, \alpha} \otimes R_{y z, \beta} .
$$

For fixed $x$ and $z$, the union, over all $\alpha$ and $\beta$, of the relations in (9) is (3), by the preliminary observation in (11). The union of all relations of the form (7) therefore coincides with the union of all relations of the form (3), and this latter union is just $U \times U$, by (5). Conclusion: the equation in (6) holds in $\mathfrak{C}[\mathcal{F}]$ for all $y$ in $I$, as was to be shown.

We postpone the proof of the reverse implication of the theorem until after the next theorem.

It turns out that every full coset relation algebra can be decomposed into the direct product of simple, full coset relation algebras, or equivalently, full coset relation algebras on simple frames. We sketch briefly how this decomposition may be accomplished. Given an arbitrary coset semi-frame

$$
\left.\mathcal{F}=\left(\left\langle G_{x}: x \in I\right\rangle,\left\langle\varphi_{x y}:(x, y) \in \mathcal{E}\right\rangle,\right\rangle,\left\langle C_{x y z}:(x, y, z) \in \mathcal{E}_{3}\right\rangle\right),
$$

consider an equivalence class $J$ of the isomorphism index set $\mathcal{E}$. The universal relation $J \times J$ on $J$ is a subrelation of $\mathcal{E}$, and in fact it is a maximal connected component of $\mathcal{E}$ in the graph-theoretic sense of the word. The restriction of $\mathcal{F}$ to $J$ is defined to be the group triple

$$
\left.\mathcal{F}_{J}=\left(\left\langle G_{x}: x \in J\right\rangle,\left\langle\varphi_{x y}:(x, y) \in J \times J\right\rangle,\right\rangle,\left\langle C_{x y z}:(x, y, z) \in J \times J \times J\right\rangle\right)
$$

Each such restriction of $\mathcal{F}$ to an equivalence class of the index set $\mathcal{E}$ inherits the coset semi-frame properties of $\mathcal{F}$, and is therefore a simple semi-frame. Call these restrictions the components of $\mathcal{F}$. Clearly, $\mathcal{F}$ is the disjoint union of its components in the sense that the group system, the isomorphism system, and the coset system of $\mathcal{F}$ are obtained by respectively forming the unions of the group systems, the isomorphism systems, and the coset systems of the components of $\mathcal{F}$. It is also easy 
to see that $\mathcal{F}$ satisfies the coset conditions if and only if each component satisfies the coset conditions, because these conditions are formulated only for cosets $C_{x y z}$ such that the elements $x, y$, and $z$ all belong to the same equivalence class of $\mathcal{E}$.

If $\mathcal{F}$ is a semi-frame satisfying the coset conditions, then so is each component $\mathcal{F}_{J}$, and consequently $\mathfrak{C}\left[\mathcal{F}_{J}\right]$ is a full coset relation algebra that is simple, with base set and unit

$$
U_{J}=\bigcup_{x \in J} G_{x} \quad \text { and } \quad E_{j}=U_{J} \times U_{J}
$$

respectively. The coset relation algebra $\mathfrak{C}[\mathcal{F}]$ is isomorphic to the direct product of the simple coset relation algebras $\mathfrak{C}\left[\mathcal{F}_{J}\right]$ constructed from the components of $\mathcal{F}$ (so $J$ varies over the equivalence classes of $\mathcal{E}$ ). In fact, if internal direct products are used instead of Cartesian direct products, then $\mathfrak{C}[\mathcal{F}]$ is actually equal to the internal direct product of the full coset relation algebras constructed from its component semi-frames.

Theorem 6.2 (Decomposition Theorem). Every full coset relation algebra is isomorphic to a direct product of full coset relation algebras on simple frames.

The details of the proof of this theorem are left to the reader.

Return now to the proof of the reverse implication in Theorem 6.1. Assume that the given semi-frame $\mathcal{F}$ is not simple. If the group index set $I$ is empty, then the base set $U$ is also empty, and in this case $\mathfrak{C}[\mathcal{F}]$ is a one-element relation algebra with the empty relation as its only element. In particular, $\mathfrak{C}[\mathcal{F}]$ is not simple. On the other hand, if the group index set $I$ is non-empty, then the isomorphism index set $\mathcal{E}$ has at least two equivalence classes, by the definition of a simple semi-frame. The coset relation algebra $\mathfrak{C}[\mathcal{F}]$ is isomorphic to the direct product of the coset relation algebras on the component semi-frames of $\mathcal{F}$, by Decomposition Theorem 6.2 and there are at least two such components. Each of these components is a simple semi-frame that satisfies the coset conditions, so the corresponding coset relation algebra must be simple, by the first part of the proof of Theorem 6.1. It follows that $\mathfrak{C}[\mathcal{F}]$ is isomorphic to a direct product of at least two simple relation algebras, so $\mathfrak{C}[\mathcal{F}]$ cannot be simple. For example, the projection of $\mathfrak{C}[\mathcal{F}]$ onto one of the factor algebras is a non-constant homomorphism that is not injective.

\section{REFERENCES}

[1] Andréka, H. and Givant, S., A representation theorem for measurable relation algebras with cyclic groups, Transactions of the American Mathematical Society, to appear.

[2] Givant, S., Introduction to relation algebras, Springer International Publishing AG, 2017, xxxii + 572 pp.

[3] Givant, S., Advanced topics in relation algebras, Springer International Publishing AG, 2017, xix + 605 pp.

[4] Givant, S. Relation algebras and groups, Algebra Universalis, to appear.

[5] Givant, S. and Andréka, H., Groups and algebras of binary relations, The Bulletin of Symbolic Logic, 8 (2002), pp. 38-64.

[6] Givant, S. and Andréka, H., A representation theorem for measurable relation algebras, submitted for publication.

[7] Hirsch, R. and Hodkinson, I., Relation algebras by games, Studies in Logic and the Foundations of Mathematics, vol. 147, Elsevier Science, North-Holland Publishing Company, Amsterdam, 2002, 712 pp.

[8] Maddux, R. D., Pair-dense relation algebras, Transactions of the American Mathematical Society, 328 (1991), pp. 83-131.

[9] Maddux, R. D., Relation algebras, Studies in Logic and the Foundations of Mathematics, vol. 150, Elsevier Science, North-Holland Publishing Company, Amsterdam, 2006, xxvi + $731 \mathrm{pp}$. 
Hajnal Andréka, Alfréd Rényi Institute of Mathematics, Hungarian Academy of Sciences, ReÁltanoda utca 13-15, Budapest, 1053 Hungary

E-mail address: andreka.hajnal@renyi.mta.hu

Steven Givant, Mills College, 5000 MacArthur Boulevard, Oakland, CA 94613

E-mail address: givant@mills.edu 